\title{
GAUSSIAN PROCESS LINKING FUnCTIONS FOR MIND, BRAIN AND BEHAVIOR
}

\section{A PREPRINT}

\author{
Giwon Bahg \\ Department of Psychology \\ The Ohio State University \\ Columbus, $\mathrm{OH} 43210$ \\ bahg.1.osu@gmail.com
}

\author{
Daniel G. Evans \\ Department of Psychology \\ The Ohio State University \\ Columbus, $\mathrm{OH} 43210$ \\ evans.2307@osu.edu \\ Brandon M. Turner \\ Department of Psychology \\ The Ohio State University \\ Columbus, OH 43210 \\ turner.826@gmail.com
}

\author{
Matthew Galdo \\ Department of Psychology \\ The Ohio State University \\ Columbus, $\mathrm{OH} 43210$ \\ brendan.m.galdo@gmail.com
}

November 13, 2019

\begin{abstract}
The link between mind, brain, and behavior has mystified philosophers and scientists for millennia. Recent progress has been made by forming statistical associations between manifest variables of the brain (e.g., EEG, fMRI) and manifest variables of behavior (e.g., response times, accuracy) through hierarchical latent variable models [1]. Within this framework, one can make inferences about the mind in a statistically principled way, such that complex patterns of brain-behavior associations drive the inference procedure. However, previous approaches were limited in the flexibility of the linking function, which has proven prohibitive for understanding the complex dynamics exhibited by the brain. In this article, we propose a data-driven, non-parametric approach that allows complex linking functions to emerge from fitting a hierarchical latent representation of the mind to multivariate, multimodal data. Furthermore, to enforce biological plausibility, we impose both spatial and temporal structure so that the types of realizable system dynamics are constrained. To illustrate the benefits of our approach, we investigate the model's performance in a simulation study and apply it to experimental data. In the simulation study, we verify that the model can be accurately fit to simulated data, and latent dynamics can be well recovered. In an experimental application, we simultaneously fit the model to fMRI and behavioral data from a continuous motion tracking task. We show that the model accurately recovers both neural and behavioral data, and reveals interesting latent cognitive dynamics. Finally, we provide a test of the model's generalizability by assessing its predictive accuracy in a cross-validation test.
\end{abstract}

Keywords Model-based cognitive neuroscience $\cdot$ Joint modeling $\cdot$ Gaussian process $\cdot$ Dimensionality reduction

\section{Introduction}

In the present technological state, the mind remains a latent construct that cannot be directly observed. However, clever experimental designs as well as technological advancements have enabled the collection of many important manifest variables such as response time, the blood oxygenated level dependent (BOLD) response in functional magnetic resonance imaging (fMRI), and the electroencephalogram. Each of these measures ostensibly reflect signatures of mental operations, potentially revealing insight into latent cognitive dynamics. Despite these innovations, the key to understanding what the mind is and ultimately how it produces complex patterns of thought and decisions, lies in our 


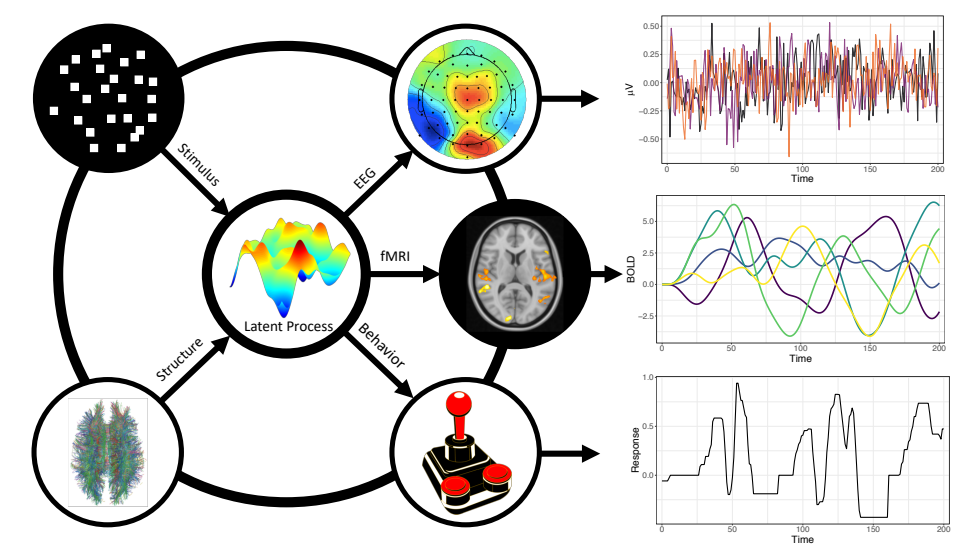

Figure 1: Framework for Connecting Mind and Brain. Schematic of the joint modeling framework, where experimental and structural information specify the structure of a generative model of brain function. The generative model is used to jointly explain all available manifest variables such as response times, blood oxygenated level dependent response, or electroencephalogram activity. In the present article, a latent Gaussian process is used to link the manifest variables, enabling the most plausible linking function to emerge directly from data.

ability to explain the structure between manifest variables of the brain, and manifest variables of behavior. The set of possible links connecting these variables are known as linking propositions [2-5].

Linking propositions have been a productive route forward because they facilitate quantitative assessments of the contribution of physiological variables to psychological processes. For example, Teller [3] devised a set of linking propositions by specifying logical relations among physiological variables and psychological states. Teller's families of linking propositions were specified to be axiomatic in the sense that they relied on strict equality statements, which are impossible to satisfy in the context of measurement noise [4]. Recognizing these practical limitations, Schall [4] developed the concept of statistical linking functions, where probability distributions could replace axiomatic statements to accommodate the uncertainty associated with manifest variables. Finally, Forstmann and colleagues [6.7| concretized statistical linking functions by performing null hypothesis tests among patterns of neural data and the parameters of computational models. Later, Forstmann and colleagues further articulated the concept of reciprocity in linking functions, where latent representations of the mind (i.e., as instantiated by computational models) could inform the analysis of manifest variables of brain and behavior [8].

There now exist many techniques for imposing linking functions, varying along several dimensions such as the manner in which they impose directionality (e.g., neural data constrains a computational model, computational model guides analysis of neural data, or equal reciprocity between all variables), the manner in which the mind is represented (e.g., a latent representation or set of transformation equations), and the complexity of their implementation [9]. One promising framework that exploits the aforementioned concepts of statistical linking and reciprocity is the joint modeling framework [10] shown in Figure 1. Constraints about the manifest variables, such as structural connectivity or experimental design, are used to specify the structure of a generative model. In turn, the generative model is specified to simultaneously describe all available manifest variables, regardless of their modality. The manner in which the latent states are connected to the manifest variables forms the linking function. By fitting the model to data using hierarchical Bayesian methods [11], one can infer the most plausible set of linking functions that connect the manifest variables.

Although joint models have proven effective in linking subject- (e.g., [10]), modality- (e.g., combining fMRI and EEG; [12]) and trial-specific (e.g., single-trial BOLD response; [13-15]) information, so far all joint modeling applications have imposed a linearity assumption among the latent and manifest variables. While linearity may be a reasonable assumption in some cases, because the brain is highly dynamic [16], this assumption is surely violated in many realistic settings. In this article, we expand the general structure used in joint modeling to enable any conceivable form of linking function to emerge directly from data.

\section{Gaussian Process as a Linking Function}

The new approach, discussed here, uses a Gaussian process (GP) to model the latent variables that represent the cognitive states underlying observed neural and behavioral data. Our Gaussian process joint modeling (GPJM) framework can be 
viewed as a temporal and nonparametric extension of the covariance-based linking function [9, 15]. In this section, we provide a brief conceptual introduction to Gaussian processes and the GPJM.

\subsection{Gaussian Process Regression}

Our goal is to find a function that best describes the data assuming Gaussian noise:

$$
y=\mathbf{f}(\mathbf{x})+\epsilon \text { where } \epsilon \sim \mathcal{N}\left(0, \sigma_{\epsilon}^{2}\right)
$$

where $\mathcal{N}\left(\mu, \sigma_{\epsilon}^{2}\right)$ is a normal distribution with mean $\mu$ and covariance $\sigma_{\epsilon}^{2}$. Typical linear regression methods model the function $\mathbf{f}$ as a sum of pre-specified bases $X$ weighted by regression coefficients $\beta$, e.g., $\mathbf{f}=X \beta$.

On the contrary, Gaussian process regression directly models the function $\mathbf{f}$ as a sample from a distribution over functions. Specifically, the distribution over functions is assumed to be a multivariate Gaussian distribution with mean $\mathbf{m}$ and covariance matrix $K$, both defined as a function of input $\mathbf{x}$ [17, 18]. Given an input vector $\mathbf{x}=\left(x_{1}, \cdots, x_{k}\right)^{\prime}$, a function $\mathbf{f}$ is modeled as a Gaussian process by

$$
\begin{aligned}
\mathbf{f}(\mathbf{x}) & \sim \mathcal{N}\left(\mathbf{m}(\mathbf{x}), K_{x x}\right) \\
\text { where } K_{x x} & :=\left[k\left(x_{i}, x_{j}\right)\right]_{i j} \\
& =\mathbb{E}\left[\left\{\mathbf{f}\left(x_{i}\right)-\mathbf{m}\left(x_{i}\right)\right\}\left\{\mathbf{f}\left(x_{j}\right)-\mathbf{m}\left(x_{j}\right)\right\}\right]_{i j} .
\end{aligned}
$$

Here the covariance matrix $K_{x x}$ is called the kernel or covariance function.

In a Gaussian process with noisy observations assuming Gaussian-distributed error, mean predictions about unobserved input $X^{*}$, denoted $\overline{\mathbf{f}}^{*}$, are modeled using a joint multivariate normal distribution prior with the kernel $K$, the observed input $X$ and output $\mathbf{y}$ [17, 18]:

$$
\left[\begin{array}{c}
\mathbf{y} \\
\mathbf{f}^{*}
\end{array}\right] \sim\left(\mathbf{0},\left[\begin{array}{cc}
K(X, X)+\sigma_{\epsilon}^{2} I & K\left(X, X^{*}\right) \\
K\left(X^{*}, X\right) & K\left(X^{*}, X^{*}\right)
\end{array}\right]\right)
$$

The predictive mean $\overline{\mathbf{f}}^{*}$ and predictive covariance matrix $\Sigma^{*}$ are analytically derived as follows:

$$
\begin{aligned}
\overline{\mathbf{f}}^{*}= & K\left(X^{*}, X\right)\left[K(X, X)+\sigma_{\epsilon}^{2} I\right]^{-1} \mathbf{y} \\
\Sigma^{*}= & K\left(X^{*}, X^{*}\right) \\
& -K\left(X^{*}, X\right)\left[K(X, X)+\sigma_{\epsilon}^{2} I\right]^{-1} K\left(X, X^{*}\right)
\end{aligned}
$$

where $K(M, N)=\left[k\left(m_{i}, n_{j}\right)\right]_{i j}$ is the kernel whose elements are determined by the $i$-th row of matrix $M$ (denoted $m_{i}$ ) and the $j$-th row of matrix $N$ (denoted $n_{j}$ ). This fact suggests that appropriate choice of kernels is important for the performance of the model.

Some popular kernels are Matérn class, radial basis function (RBF), and rational quadratic (RQ) kernels. Each kernel generates different types of functions based on their functional form. For example, Matern $1 / 2$ kernels generate nondifferentiable functions, whereas RBF kernels generate infinitely differentiable, smooth functions. Hyperparameters (which typically include a scaling factor often called 'variance') of the kernel also affect the characteristic evaluating covariance across input points 1 In particular, all the kernels introduced above have a hyperparameter called 'lengthscale' that scales the distance between two points. This hyperparameter plays an important role in determining the influence or 'relevance' of each latent dimension in generating data, which will be discussed later.

\subsection{Gaussian Process Joint Models}

GPJMs inherit the motivation of Bayesian joint modeling approaches accounting for brain-behavior reciprocity [10 12 - 15, 20]: Imposing common statistical constraints for both neural and behavioral data when informing theoretical and mechanistic explanations of cognitive processes. However, instead of summarizing the relationship between neural and behavioral measures by collapsing across temporal information, the GPJM framework aims to learn latent temporal dynamics that simultaneously govern both neural and behavioral data. The GPJM framework is based on Gaussian process latent variable models (GPLVMs) and their hierarchical extensions [21-24], which pursue commonly shared latent representations across simultaneous observations from multiple measurement sources. In a previous application, a GPLVM has been used successfully to model the latent structure embedded in multivariate neural spike train data [25].

\footnotetext{
${ }^{1}$ To avoid confusion between the kernel variance and the variance of the noisy observations, we will refer to the latter as 'observation noise' and the former as 'variance'.
} 


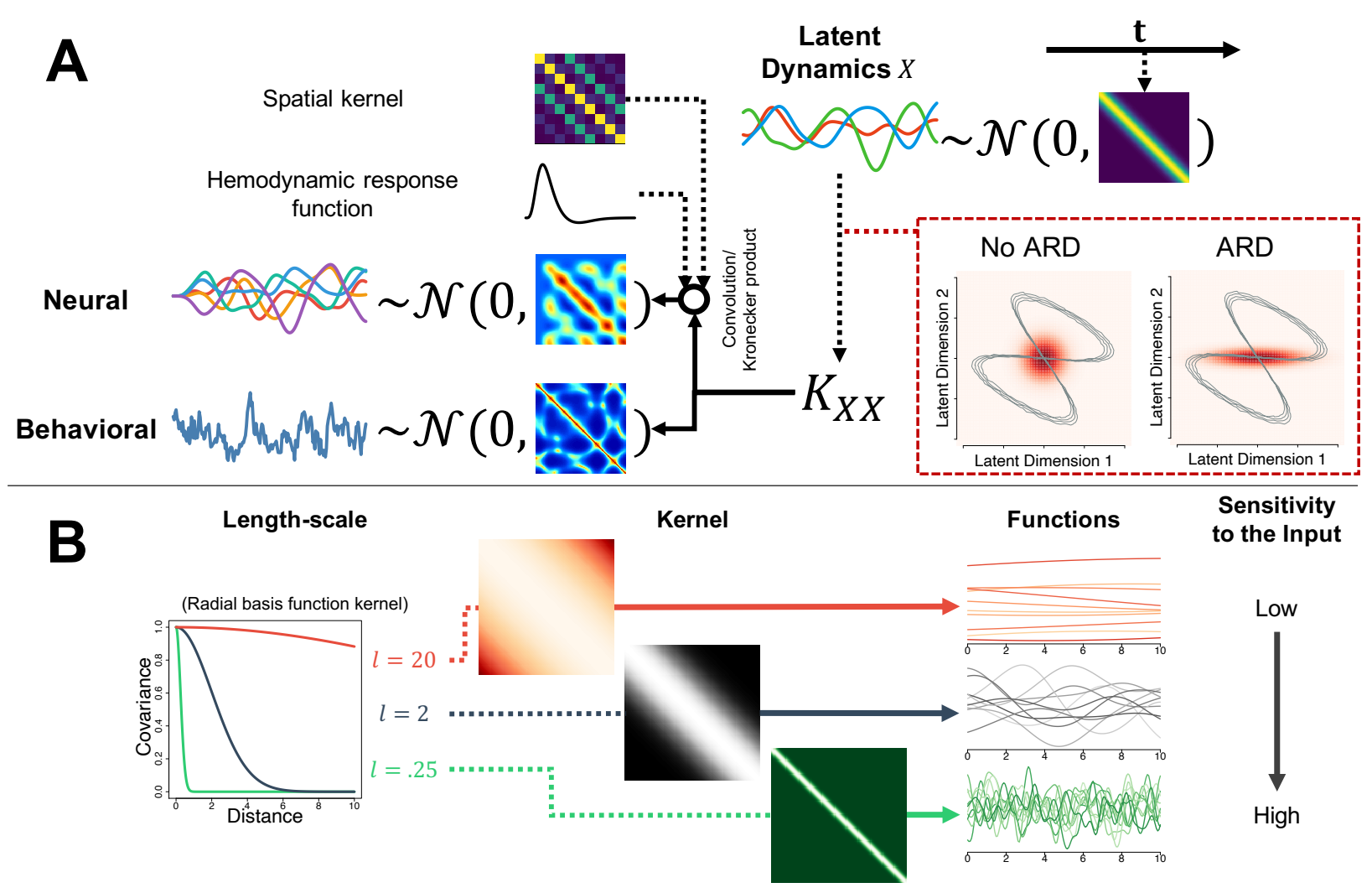

Figure 2: The Structure of Gaussian Process Joint Models. (A) The generative mechanism of the Gaussian process joint models. Given the time vector $\mathbf{t}$, the linking function describing latent cognitive states is modeled by Gaussian processes with a temporal kernel as a function of $\mathbf{t}$ (top right). The linking function, denoted $X$, generates a cognitive state kernel $K_{X X}$ and is introduced as the covariance function of Gaussian processes modeling neural and behavioral data (bottom left). Depending on the nature of neural data, $K_{X X}$ can be applied after transformation (e.g., convolution with a hemodynamic response function for fMRI data). In this study, we use automatic relevance determination (ARD; [19]) so that each dimension of the latent cognitive state can contribute to the generation of data with different weights of influence. Here, kernel parameters capture sensitivity of data to each latent cognitive dimension using dimension-wise length-scale parameters (bottom right). (B) Details of the ARD-based feature selection mechanism. A small length-scale parameter means that the covariance between two input points are more penalized by their distance and therefore differences in the input values are interpreted more importantly (bottom). Meanwhile, a greater length-scale parameter can make even distant input points covary and therefore minor differences in the input are treated negligible (top). fMRI = functional magnetic resonance imaging.

Joint models of neural and behavioral data consist of three components: a neural submodel, a behavioral submodel, and a linking function. As illustrated in Figure 2, in GPJM a latent variable $X$ with a GP prior enables a linking function that constrains the generative process for neural and behavioral data:

$$
X \sim \mathcal{N}\left(\mathbf{0}, K_{t t}\right)
$$

where $\mathbf{0}$ is a column vector with all zeros, and $K_{t t}$ is a kernel defined by time points. For example, given a time vector $\mathbf{t}=\left(t_{1}, \cdots, t_{T}\right)^{\prime}$ of length $T$, a radial basis function (RBF) kernel is defined as

$$
K_{t t}=\left[k_{t}\left(t_{i}, t_{j}\right)\right]_{i j}:=\left[s_{t}^{2} \exp \left(-\frac{\left(t_{i}-t_{j}\right)^{2}}{2 l_{t}^{2}}\right)\right]_{i j}
$$

where $i, j \in\{1,2, \cdots, T\}$, and $s_{t}$ and $l_{t}$ are the hyperparameters of the RBF kernel (variance and length-scale, respectively). Here, we assume that the latent cognitive dynamics are noise-free.

Given the latent cognitive dynamics $X$, a neural submodel describes the relationship between the latent dynamics at the hyper level and the neural data. Specifically, a neural submodel of the GPJM assumes that the neural data are described 
by a Gaussian process with a RBF kernel $K_{X X(N)}$ based on $X$ :

$$
\begin{aligned}
Y_{N} & \sim \mathcal{N}\left(\mathbf{0}, K_{X X(N)}+\sigma_{N}^{2} I\right), \\
K_{X X(N)} & =\left[k_{N}\left(x_{i}, x_{j}\right)\right]_{i j}:=\left[s_{N}^{2} \exp \left(-\frac{\left(x_{i}-x_{j}\right)^{2}}{2 l_{N}^{2}}\right)\right]_{i j}
\end{aligned}
$$

where $x_{k}(k \in\{1, \cdots, T\})$ indicates the $k$-th row of $X$, and $s_{N}$ and $l_{N}$ are the hyperparameters of the RBF kernel (variance and length-scale, respectively). Note that the temporal dynamics kernel $K_{X X(N)}$ requires additional adjustments depending on the nature of the neural data (see "GPJM and Technical Issues in Brain-Behavior Modeling"). To capture the residual noise in $Y_{N}$, a noise parameter $\sigma_{N}^{2}$ scales an identity matrix $I$.

A behavioral submodel provides a formal description of how cognitive dynamics produce behavioral responses. In typical joint modeling applications, cognitive models are used to describe the computations underlying decision processes. In this particular study, because there are no suitable computational models for our task, we will use a Gaussian process as a statistical model to describe continuous mouse trajectories, derived from the cognitive dynamics. For our purposes, the behavioral submodel incorporates the temporal dynamics using a Matérn $1 / 2$ kernel:

$$
\begin{aligned}
Y_{B} & \sim \mathcal{N}\left(\mathbf{0}, K_{X X(B)}+\sigma_{B}^{2} I\right), \\
K_{X X(B)} & =\left[k_{B}\left(x_{i}, x_{j}\right)\right]_{i j}:=\left[s_{B}^{2} \exp \left(-\frac{\left\|x_{i}-x_{j}\right\|}{l_{B}}\right)\right]_{i j}
\end{aligned}
$$

where llall means the norm or length of the vector $a$, and $s_{B}$ and $l_{B}$ are the hyperparameters of the Matérn $1 / 2$ kernel (variance and length-scale, respectively). As in the neural data, residual error in $Y_{B}$ is accounted for by the noise parameter $\sigma_{B}^{2}$.

In Equations 3 and 4, length-scale parameters $l_{N}$ and $l_{B}$ scale the distance between pairs of input points and control their covariance. Figure $2 \mathrm{~B}$ illustrates the role of length-scale parameters: when the length-scale parameter is large, the covariance between two input points is weakly penalized by the distance between them. Hence, large length-scale parameters allow distant pairs of points to covary and makes sampled functions relatively constant across input values (see Figure 23). By contrast, when the length-scale parameter is small, only nearby input points can covary whereas distal points are uncorrelated. Automatic relevance determination (ARD; [19]) extends this idea to each latent input by assigning one length-scale parameter per dimension. By applying ARD to the kernels of the behavioral and neural submodels, the GPJM can learn how much each dimension contributes to the data generation process; in other words, the relevance of the latent input in the context of the data (lower right, Figure 2A).

\subsection{GPJM and Technical Issues in Brain-Behavior Modeling}

One of the major issues in joint modeling approaches is the mismatch of the task-related timeline between neural and behavioral data. This problem is more salient when applying the model to data from fMRI experiments due to the hemodynamic lag between the neural response and the experimental event. Hemodynamic activities are characterized by a delayed increase in response, attaining a maximum value approximately 5-6 seconds after an event (e.g., stimulus presentation), a slow decrease in the neural response, and finally a drop in the neural response below the baseline, followed by a recovery to a baseline state. Because of this temporal profile, the hemodynamic lag obscures precise temporal profiles of neural activity.

The interaction between underlying neural activities and hemodynamics in the brain is typically modeled by convolving neural activations with a hemodynamic response function (HRF), which facilitates general linear model analyses of fMRI data [26]. Early joint modeling approaches have taken advantage of this assumption, and used event-wise neural activation estimates [27] for trial-level summaries of neural activity [13] 14].

In the Gaussian process framework, one way to enforce temporally-lagged, convolved neural signals is to convolve the GP kernel with a secondary function - a HRF in our case. This approach is justified from the fact that convolution of a Gaussian process with another function is another Gaussian process [28-30]. In the GPJM, we use a kernel convolved with an HRF as a key component of the neural submodel. Here, the latent cognitive dynamics are expected to be relatively well aligned to the real-time neural activity because the resulting kernel instantiates hemodynamic lag.

To instantiate hemodynamic lag, we use the canonical double-gamma HRF:

$$
h(t)=\frac{t^{a_{1}-1} b_{1}^{a_{1}} \exp \left(-b_{1} t\right)}{\Gamma\left(a_{1}\right)}-c \frac{t^{a_{2}-1} b_{2}^{a_{2}} \exp \left(-b_{2} t\right)}{\Gamma\left(a_{2}\right)}
$$

where $t$ represents time, and $\Gamma(x)=(x-1)$ ! is the gamma function. Although estimating shape parameters of the HRF is theoretically possible, here we rely on the canonical shape by fixing the shape parameters to values based on previous work by Glover [31] and commonly used in brain analysis software packages: $a_{1}=6, a_{2}=16, b_{1}=b_{2}=1, c=1 / 6$. 

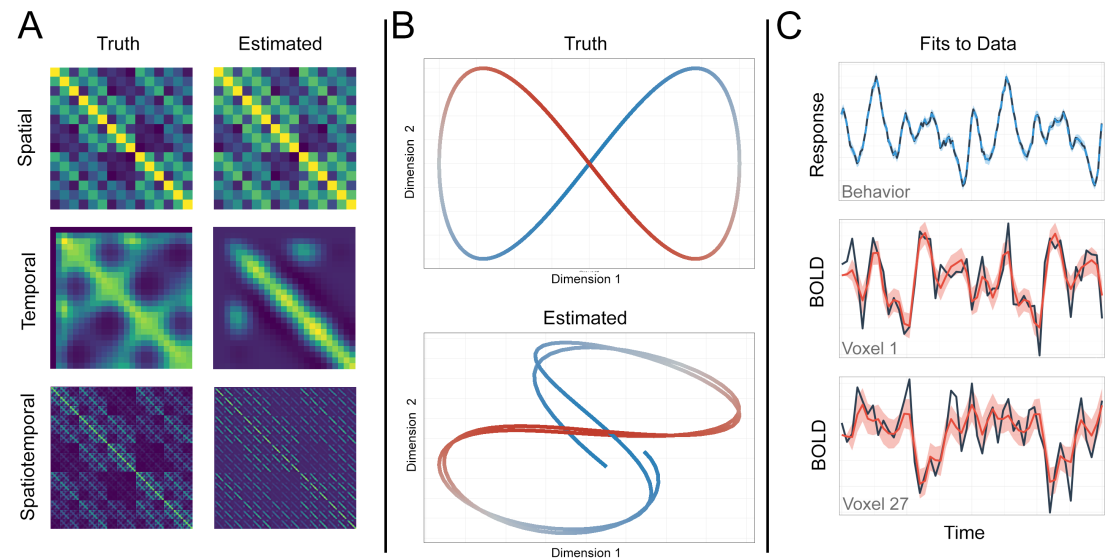

Figure 3: Simulation Results. (A) Spatial, latent temporal, and Kronecker-product kernels used to generate synthetic data (left) and those estimated from fitting the model (right) are shown. Only a subsection of each kernel is presented here for visual clarity. (B) Latent representations used to generate the data (top) and estimated from fitting the model to data (bottom). Each representation is shown as a two-dimensional trajectory, arbitrarily color-coded according to moments within one of two cycles. (C) Data (black) and model predictions (blue behavioral; red: neural) are shown for behavioral (top) and two representative neural (middle, bottom) time series. For the model predictions, solid lines are the mean prediction and the surrounding bands are $95 \%$ credible interval.

Another methodological issue in joint modeling approaches is applying plausible spatiotemporal constraints on the neural data. For computational convenience, previous joint modeling applications have assumed statistical independence between regions of interest (ROIs; e.g., [12, 13]), which has clear limitations given the structural and functional connectivity of the brain. An ideal statistical representation would incorporate both spatial (e.g., nearby and wellconnected ROIs should correlate) and temporal (e.g., activity at one time point in one ROI should affect activity at later time points in other, "downstream" ROIs) dependencies among ROIs. To instantiate such constraints, we will separately define kernels for space $K_{S}$ and time $K_{T}$, and combine them using the Kronecker product (e.g., [32]33]) such that

$$
\operatorname{vec}\left(Y_{N}\right) \sim \mathcal{N}\left(\mathbf{0}, K_{S} \otimes K_{T}\right)
$$

where $Y_{N}$ is a $(M \times V)$ matrix with $M$ measurements and $V$ voxels (or ROIs), vec $(M)$ represents a vectorization operator stacking columns of a matrix $M$ as a single column vector, and $\otimes$ is the Kronecker product operator. In using the Kronecker product, we are assuming that although space and time have independent influences on the neural dynamics, their conjunction can be appropriately enforced when modeling the neural data. When we cannot assume independence between the spatial and temporal aspects of our data, non-separable kernels are useful alternative options (e.g., [34]).

In the following two sections, we examine the potential of Gaussian processes as a linking function connecting neural and behavioral measures in two ways. First, we examine our ability to correctly recover the spatiotemporal dynamics in a simulated example using the Kronecker-product method. Next, we apply our GPJM to data from a real fMRI experiment in which subjects are asked to provide a continuous report of motion coherence.

\section{Simulation: The Kronecker Method for Spatiotemporal Modeling}

Before applying the GPJM to data, it is important to evaluate our ability to accurately fit data and recover latent dynamics. In particular, we examine whether it is possible to recover accurate spatiotemporal dynamics of the Kronecker-product kernel governing the distribution of behavioral and neural data. In this section, we perform a simulation study with similar structure as the experiment we report below.

The latent cognitive states $C_{i}(t)(i=\{1,2\})$ were defined by two sine waves with different frequencies:

$$
\begin{aligned}
& C_{1}(t)=\sin (t / 8), \text { and } \\
& C_{2}(t)=\sin (t / 4) .
\end{aligned}
$$

These state vectors comprised a latent state matrix $X$, and were used to generate synthetic neural and behavioral data. For the neural data, we assumed 27 adjoining voxels in a three-dimensional space, and for the behavioral data we 
assumed one joystick trajectory data vector. As in the neural (temporal) kernel $K_{X X(N)}$, we used a RBF kernel for describing the spatial covariance. Note that (1) we did not apply ARD in this simulation; and (2) the variance parameter of the spatial kernel (corresponding to $s_{N}^{2}$ in Equation 3 was fixed to one to avoid identifiability issues; specifically, this constraint is necessary because the two variance parameters corresponding to the spatial and temporal kernels cannot be distinguished after applying the Kronecker product.

Figure $3 \mathrm{~A}$ illustrates a subset of the three kernels used to generate the data (left) and estimated from the data (right): spatial (top), temporal (middle), and resulting Kronecker product (bottom). Figure $3 B$ illustrates the true latent dynamics (top) and the recovered latent dynamics (bottom) as a joint trajectory through a two dimensional space. Colors gradually changing across points specify the relative position in the cyclic temporal dynamics. Figure $3 \mathrm{C}$ shows the data (black) and model predictions (neural: red; behavioral: blue) for the behavioral data (top) and two selected voxels (middle, bottom). These plots reveal that (1) the spatiotemporal GPJM can capture the spatial relationship among voxels using the Kronecker method; and (2) the model can estimate the latent dynamics that explain the generative process of the data with high accuracy. Note that the latent dynamics do not have a naturally unique solution, and so we cannot expect our estimated latent dynamics to perfectly match. Instead, to evaluate the quality of the latent dynamics, one must comprehensively consider model fit to data and statistical structure of the latent dynamics (e.g., the frequency and cyclical information).

\section{4 fMRI Experiment}

As a proof of concept, we next apply the GPJM to experimental data from an ongoing study. In the experiment, the participant was presented with a cloud of randomly moving dots, with a subset of those dots moving in a coherent direction. The proportion of dots exhibiting coherent movement had the possibility of changing every second. The proportion of dots formed the independent variable of coherence, which was defined by nine points along a left-right axis. The participant was instructed to report the average direction of the dots from the beginning of the trial to the current point in time using a joystick.

We used the GPJM defined in the simulation study above to capture dynamics of the neural and behavioral data in our task. However, to scale the model to fit the data, we made a few simplifications of the full spatiotemporal GPJM: (1) we performed an ROI-based analysis; (2) neural data were introduced into the model after averaging signals across voxels within a set of 16 ROIs; and (3) we used only a temporal kernel rather than the full spatiotemporal Kronecker-product method. Figure 4A shows the ROIs, which were pre-selected using a general linear modeling (GLM) analysis (see the "Materials and Methods" section and Supplementary Information for full details).

\subsection{Fits to Data}

Figure 4 summarizes the results of the model fit to data. Although we tested GPJMs using three, four, five, and six latent dimensions, Figure 4 summarizes the result of the three dimensional model for visual clarity (but see Supplementary Information for the four dimensional model). Figure $4 \mathrm{~A}$ shows the ROIs that were first extracted from our GLM analysis, from which the neural time series were extracted. Figure $4 \mathrm{~B}$ shows some example neural (top; middle) and behavioral (bottom) time series data (black) along with corresponding model fits (blue: behavior; red: neural). Figure $4 \mathrm{C}$ illustrates the latent temporal dynamics that emerged in the GPJM using a three-dimensional representation. Figure 4D shows the time series information for each of the three latent dimensions. Some abnormal deviations of the latent dimensions are highlighted (gray) in Figure 4D, and these deviations have corresponding paths in Figure $4 \mathrm{C}$ (demarcated by orange dots).

\subsection{Out-of-sample validation}

Dimensionality of the latent representation used within the GPJM is crucial in accurately fitting data. Although more latent dimensions tends to fit observed data better, it may not generalize well to new data. To evaluate the generalizability of the GPJM, in this section we will perform a quantitative model comparison by evaluating how the number of latent dimensions affects generalization performance in an out-of-sample validation test.

To examine the effects of the number of latent dimensions on the GPJM's generalization performance, we will attempt to predict behavioral data from the combination of latent dynamics and temporal structure in the behavioral data.

As our modeling approach depends on the temporal dependencies of the data, typical approaches in cross-validation practices, such as excluding an entire trial (roughly corresponding to 70-80 time points), will unfairly penalize a dynamical model due to the lack of context in the structure of the data. Instead, we removed $x$ data points at random points in time from within each trial in the task, and treated this set of data points as the test data. Such a procedure 


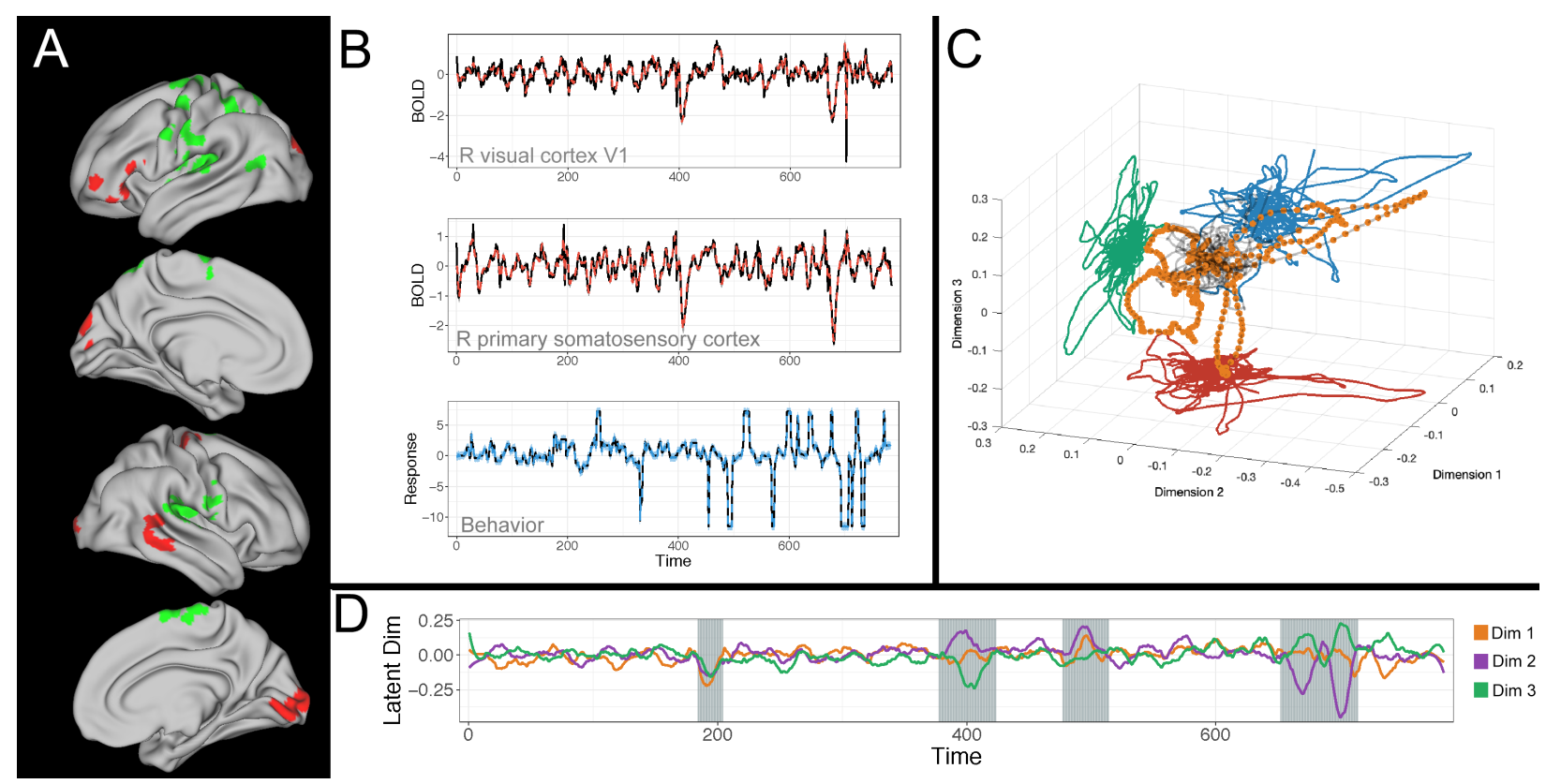

Figure 4: Fits to Experiment Data. (A) Regions of interest used within the Gaussian process joint model (GPJM). One region of interest located in cerebellum is not shown here. (B) Selected time series data from neural (top, middle) and behavioral (bottom) data, along with model fits (neural: red; behavioral: blue), along with $95 \%$ credible intervals. (C) A three-dimensional representation of the latent temporal dynamics emerging from the data after fitting the GPJM. (D) Temporal dynamics of the three latent dimensions extracted from the GPJM. Gray highlighted areas in (D) have corresponding time series information, demarcated by orange dots in $(\mathrm{C})$.

allows us to examine not only how the model generalizes temporal dynamics at different points in time, but also how the error in the generalization changes as a function of the length of consecutively removed time points.

Figure 5A shows the observed behavioral data (black) along with predictions from a four-dimensional GPJM (red lines). The blue vertical lines designate the points in time that designated the first moment from which the $n$ consecutive points were removed, creating the test set. Figure $5 \mathrm{~B}$ shows a zoomed in version of Figure $5 \mathrm{~A}$ for each of the four withheld-data conditions, where the observed behavioral data are shown in black, and the model predictions are shown in red. Even when zooming in, the model provides a reasonably good generalization to the true time series, although the accuracy of the generalization is noticeably worse as the number of withheld data points are increased.

To quantitatively evaluate the generalization performance, we used log posterior predictive density (PPD) and root mean squared error (RMSE). Figure $5 \mathrm{C}$ summarizes the predictive performance metrics across different model variants and sizes of the test data set. The log PPD (top) consistently supports the three-dimensional latent space. However, this performance is achieved by covering biased predictions with a greater margin of predictive uncertainty. The interpretation of the RMSE makes this issue clear - the RMSE of the three-dimensional model variant keeps increasing according to the size of the test data set, whereas the four-dimensional model variant tends to perform better than other model variants on average. The models with the five- and six-dimensional latent space were neither supported by log PPD nor providing accurate predictions consistently, suggesting that these model variants were overfitting the data.

\section{Discussion}

In this article, we extended an existing framework for connecting mind, brain and behavior by specifying Gaussian process linking functions for the dynamics of both latent and manifest variables. This extension allows a few key advantages that will be important in the field of model-based cognitive neuroscience [5, 8, 9, 35]. First, our approach inherits all the advantages that a hierarchical structure provides, such as statistical reciprocity through conditionally independent variables within a global model, and flexibly accounting for missing observations [1, 10, 36]. Second, the model's representation of the mind is a latent dynamical system. Because the mind is a latent construct, we believe it is best specified as a set of conditionally independent variables that can be estimated, rather than as a transformation of either neural or behavioral data (see [9] for discussions). Third, the representations of all variables within the GPJM are 


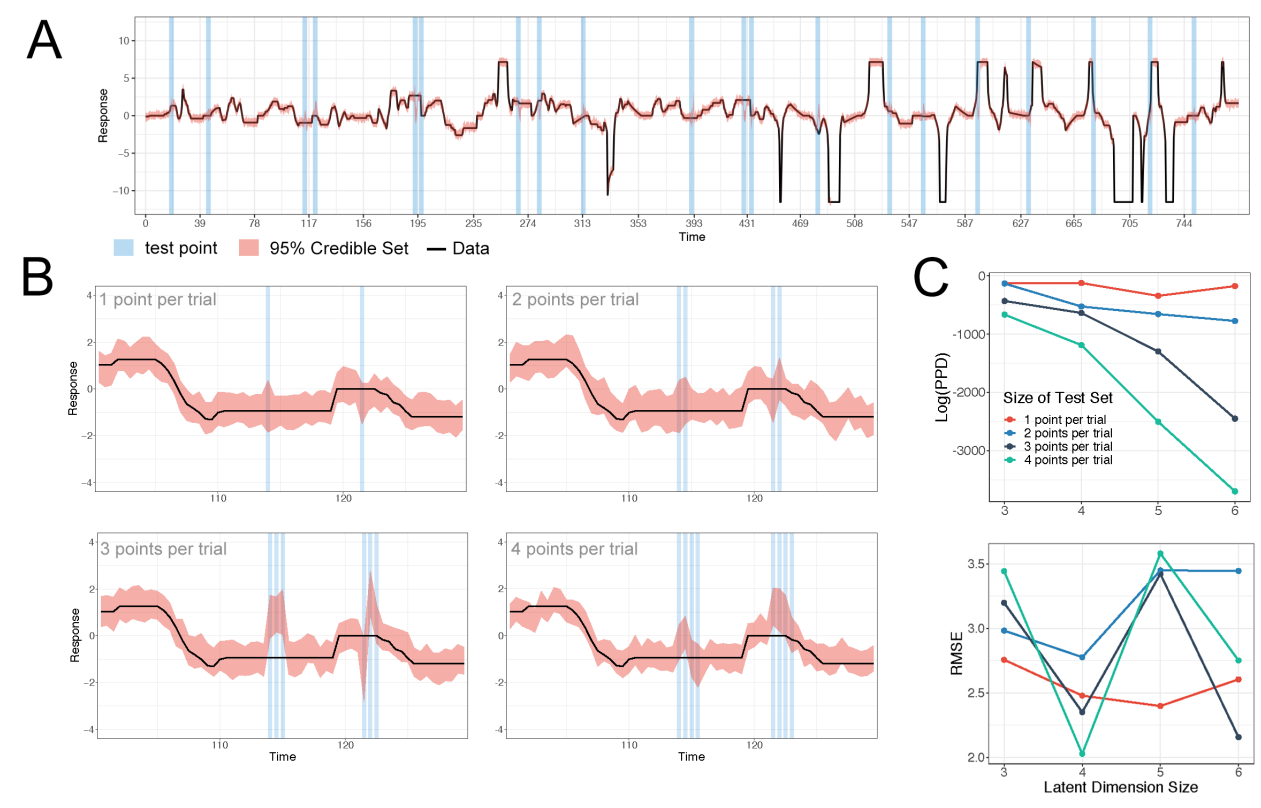

Figure 5: Out-of-sample Validation. (A) Complete time series of a participant's behavioral response (black) along with $95 \%$ credible interval of predictions from the four-dimensional model (red). The $x$-axis ticks indicate fixation onset for each trial and blue highlighted regions show the time points selected as test data for the largest set size (i.e., four points per trial). (B) Magnified view of panel A, separated by the number of consecutive test points. As the number of consecutively removed points increases, the accuracy of the model decreases. (C) The top-row and bottom-row plots show log posterior predictive densities (PPD) and root mean squared errors (RMSE) of the mean predictions from the tested models, respectively. The $x$-axis represents the dimensionality of the model and colors indicate the size of the test set.

dynamic, allowing us to infer periodic changes at both local and global time scales. Fourth, the GPJM can be specified to allow for both spatial and temporal structure through the Kronecker product. The fusion of space and time in the latent dynamics allow us to appreciate the spatiotemporal constraints that any biological system must obey.

We have only begun to investigate the advantages provided by GPJM, and so at present, there remain a number of important aspects of this innovation that merit further investigation. First, strategies for interpreting the representation inferred from the GPJM's fit to data will be important for understanding the cognitive dynamics that emerge from the model. In the simulation study, although the latent dynamics shown in Figure $3 \mathrm{~B}$ separated two cognitive states from one another, there were other estimated dynamics that were not an essential part of the ground truth. Although complex and ambiguous representations often emerge in many other machine learning applications, the field will require some type of guideline for interpretation of these cognitive dynamics if GPJMs are to advance our understanding of the connections between mind, brain and behavior.

Another limitation is the resolution of the data in constraining the GPJM. Although our experimental application used a continuous motion tracking task, most experiments in psychology and cognitive science use discrete, "event related" designs rather than block-type manipulations. Although GPJM could be used in a similar way as earlier joint modeling work [13] to exploit trial-to-trial changes in cognitive states (e.g., attention), additional theoretical innovation is needed to account for changes in cognitive states between two stimulus presentations.

From our perspective, the biggest challenge facing GPJMs is computation. To fully capitalize on all the complexities that GPJMs offer, computational solutions will be needed to increase the scalability of our approach to full-brain, spatiotemporal investigations. Although there have been attempts to increase the scalability of joint models [12, 14] and Gaussian processes, many interesting opportunities are just out of reach. For example, hierarchical extensions for voxel-wise or group-level modeling are obvious next steps, but they are currently computationally infeasible. Alternatively, two promising directions are sparse GP approximations and variational inference (e.g., [24, 37, 38]), but the tradeoffs of these approximations have yet to be well studied. For now, we propose the GPJM for its theoretical advantages, and save computational innovations for future investigations. 
One final limitation worth acknowledging is the GPJM's stationarity assumption for latent cognitive processes throughout each block. Although stationarity provides clear computational advantages, it remains an open question as to whether or not this assumption functions as a good approximation of reality. In many neural time series investigations, timefrequency analysis methods such as wavelet convolution are commonly applied to avoid the pitfalls of assuming stationarity, and further work is needed to assess the effects of stationarity in GPJM applications (e.g., [39]).

\section{Conclusion}

In this article, we have laid the statistical groundwork that will allow data to inform the links among mind, brain, and behavior. At their core, Gaussian process joint models rely on Gaussian processes (GP) that fuse temporal and spatial information, allowing complex latent dynamics to emerge from the intrinsic biological dynamics of the brain. We view the GP structure as one new way to alleviate the burden of specifying the particular shape of the linking function; instead, by specifying a flexible, nonparametric functional form, the shape of the linking function can emerge directly from data.

\section{Acknowledgment}

The authors would like to thank Michael Shvartsman for helpful discussions that motivated the present research. This research was supported by a CAREER award from the National Science Foundation (BMT). 


\section{Materials and Methods}

\subsection{Model Implementation and Training}

The model was implemented using GPflow [40], a Gaussian process modeling library based on TensorFlow [41]. The linking function and neural submodel used $\mathrm{RBF}$ kernels, whereas the behavioral submodel used a Matérn 1/2 Kernel. Although we assumed that the linking function should be free from observation noise, we had to add a small fixed noise parameter $\left(\sigma_{t}^{2}=10^{-6}\right)$ because GPflow does not support the noise-free assumption.

For efficient training, the model initialized a $k$-dimensional latent variable using the first $k$ principal components estimated from the neural data, following the suggestion from [23]. To match the number of time points between the PCs and behavioral data, we performed PCA after interpolating the neural data at all missing observation points.

For the simulation, we used L-BFGS-B [42] to obtain MAP estimates of the model parameters. Following the default setting of GPflow, optimization steps were limited to 1000 iterations at maximum. For the fMRI experiment, we used a stochastic optimization algorithm Adam [43]. The default learning rate recommended by the authors $(\alpha=0.001)$ was too small for efficient model fitting. To speed up the initial learning phase, we first initialized the learning rate parameter as $\alpha_{0}=0.20$ for fitting to the complete data and $\alpha_{0}=0.25$ for the cross-validation analyses. Then we updated the learning rate every 20 iterations following a decreasing function of the optimization steps: $\alpha_{t}=\alpha_{0} / \sqrt{t-(t \bmod 20)+1}$. The optimization steps were limited to 600 iterations at maximum.

\subsection{Participant}

The data presented here was acquired, after obtaining informed consent, from a 19-year-old male as part of a larger study that is ongoing. All participants in the data set have normal or corrected-to-normal vision and are self-reported to be right-handed. The study was approved by The Ohio State University's Institutional Review Board.

\subsection{Task and Stimuli}

The participant completed four blocks of a random dot motion task in which he was instructed to continuously track the direction (left or right) and the magnitude of coherence of the dots throughout each trial. Each block contained 20 trials separated by inter-trial intervals ranging from 6 to 8 seconds. Trials began with a 2-second fixation cross at the center of the screen followed by 30 seconds of the randomly moving dots stimulus centered on the screen. There were 150 dots that were each $3 \times 3$ pixels and the diameter of the entire dot cloud subtended 5.4 degrees of visual angle. The proportion of coherently moving dots ranged from -0.35 to 0.35 (possible values were $-0.35,-0.25,-0.15$, $-0.05,0,0.05,0.15,0.25,0.35$ ) where negative coherence indicates leftward motion, positive coherence indicates rightward motion, and zero indicates uniform circular noise. Coherence for each trial was initialized by randomly sampling one coherence level from the set of all possible values. This first state variable was also initialized to have an "age" of zero seconds. At each new second, a shift in the value of the coherence level was stochastically considered. Specifically, we sampled a value $x \sim U(0,1)$. If $x<1-\exp (-0.1 \times($ age $))$, the state moved to a new coherence value by randomly sampling one value from only the nearby coherence values to maximize smooth transitions; in this case, the age of the state was reset to zero. If not, the state remained the same and the age was incremented. The coherence sequences were generated prior to the experiment using SMILE (State Machine Interface Library for Experiments; https://smile-docs.readthedocs.io/en/latest/), a Python library for programming psychological experiments. Stimuli were displayed at a viewing distance of $74 \mathrm{~cm}$ on a rear-projection screen with a resolution of $1280 \times 1024$ pixels and a $60 \mathrm{~Hz}$ refresh rate. The participant used a Current Designs TETHYX HHSC-JOY-5 joystick to indicate the direction and magnitude of coherent motion by moving the joystick left or right along a continuous slider, which allowed him to monitor his responses in real time. Because the behavioral data was output as the pixel position of the slider, responses were later rescaled to be -1 to 1 with .01 precision for the neural GLM analyses and .00001 for the GPJM.

\subsection{Behavioral Data Processing}

SMILE recorded the cursor position with adaptively changing sampling rate, changing from $1 \mathrm{~Hz}$ (when the cursor does not move) to $332 \mathrm{~Hz}$. For the modeling purpose, we interpolated and downsampled the data to $2 \mathrm{~Hz}$ for the neural GLM analyses and the GPJM.

The horizontal cursor position is not appropriate to model with the assumption of Gaussian likelihood because the cursor position is bounded to the range of $[0,1280]$. To model the data using a GP with Gaussian noise, we rescaled the data to the range of $[0,1]$ and logit-transformed them. As the rescaled data points with the value of 0 and 1 cannot be logit-transformed, those points were adjusted to $10^{-5}$ and $1-10^{-5}$. 


\subsection{MRI Data Analysis}

FMRI data preprocessing and analysis was performed primarily with FEAT (FMRI Expert Analysis Tool; [44]) Version 6.00 in FSL (FMRIB's Software Library; https://fsl.fmrib.ox.ac.uk/fsl/). A typical preprocessing pipeline including motion correction, fieldmap-based EPI distortion correction, removal of non-brain structures, spatial smoothing, high-pass filtering, and normalization via grand-mean scaling was used to clean each block of the 4D task data. Additionally, the T1 image was segmented into gray matter (GM), white matter (WM), and cerebrospinal fluid (CSF). EPI data were registered first to the T1-weighted image, then transformed to standard space using the same transformation matrix acquired from registering the T1 image to standard space. See supplementary materials for complete preprocessing details.

After preprocessing, FSL's general linear model tool (FILM; [45]) was used to calculate activity estimates for the preprocessed functional data from each block. Three primary predictors were included in the model: participant responses, non-zero stimulus coherence, and zero stimulus coherence. Participant responses and stimulus coherence were rescaled such that they ranged from -1 to 1 . The predictors were convolved with a double-gamma HRF to create the main regressors. Nuisance regressors including mean WM and CSF signal as well as 24 realignment estimates (X, $\mathrm{Y}, \mathrm{Z}$, pitch, yaw, roll, and their first and second order temporal derivatives) were also added to the model to account for motion and other variance of no-interest. The temporal derivatives of all regressors in the model were also added as confounds of no-interest. Finally, the time series was prewhitened within FILM to correct for autocorrelations in the BOLD signal. Contrasts for each of the three predictors of interest were calculated as the effect of interest versus no activity (i.e., zero). Additional contrasts included non-zero coherence greater than response, non-zero coherence less than response, non-zero coherence greater than zero-coherence, and non-zero coherence less than zero-coherence.

Following the block-wise analyses, a fixed-effects analysis was conducted in FEAT to assess the average effects of each contrast collapsed across blocks. The resulting clusters of the fixed-effects analysis were thresholded at $Z \geq 2.33$ and corrected for family-wise error at $p<.05$. Unsurprisingly, many of the resulting clusters from the contrasts comparing non-zero coherence to participant response or zero-coherence shared significant overlap with clusters from the main predictors. Due to the constraints of the GPJM, we selected ROIs that did not have any spatial overlap. Thus, ROIs were defined as the clusters resulting from the contrasts of non-zero coherence versus no activity and participant response versus no activity. This yielded 16 ROIs whose mean time series from a single block served as target data for the GPJM (see "Regions of Interest" in Supplementary Information for more details). 


\section{Supplementary Information A: Methods for Model Implementation}

\subsection{Convolution Processes}

We assumed that neural data are obtained from a functional magnetic resonance imaging (fMRI) experiment. The blood oxygenated level dependent (BOLD) responses measured in the MRI scanner are dominated by hemodynamic activities in the brain, rather than reflecting neural activities as they are. From a modeling perspective, this problem is addressed by assuming that BOLD responses are the result of convolving unknown neural activities with a hemodynamic response function (HRF), which describes the temporal profile of hemodynamics in the brain.

One of the complications in our approach is that neural data are convolved signals. However, we can still rely on Gausian processes for modeling BOLD responses because a Gaussian process convolved with another function is known to be a Gaussian process [28, 30]. Here we introduce how we can convolve a latent function with a HRF based on the discussion of Álvarez and Lawrence [29]. Assume that a mean prediction of neural data $f(t)$ is a product of convolving a latent activity function $u(z)$ and $\operatorname{HRF} h$, and the observation is defined by the mean production $f$ plus observation noise $\epsilon$ :

$$
\begin{aligned}
y(t) & =f(t)+\epsilon(t) \\
& =\left\{\int_{-\infty}^{\infty} h(t-z) u(z) d z\right\}+\epsilon(t)
\end{aligned}
$$

The covariance between $y\left(t_{i}\right)$ and $y\left(t_{j}\right)$ is then

$$
\begin{aligned}
k\left(y\left(t_{i}\right), y\left(t_{j}\right)\right) & =k\left(f\left(t_{i}\right), f\left(t_{j}\right)\right)+k\left(\epsilon\left(t_{i}\right), \epsilon\left(t_{j}\right)\right) \\
& =\left\{\int_{-\infty}^{\infty} h\left(t_{i}-z\right) \int_{-\infty}^{\infty} h\left(t_{j}-z^{*}\right) k\left(u(z), u\left(z^{*}\right)\right) d z^{*} d z\right\}+k\left(\epsilon\left(t_{i}\right), \epsilon\left(t_{j}\right)\right)
\end{aligned}
$$

Here, we can use the kernel generated by the latent signal $u$ for $k\left(u(z), u\left(z^{*}\right)\right)$ if $u$ is a Gaussian process.

In our study, the observation noise was assumed to be independent (i.e., $k\left(\epsilon\left(t_{i}\right), \epsilon\left(t_{j}\right)\right)=\sigma^{2} I$ where $\sigma^{2}$ is a generic expression of the observation noise parameter). In the simulation, we added the noise after the Kronecker-product spatiotemporal kernel was defined (see "Data Generation" subsection of "Simulation: Synthetic Data Generation").

\subsection{Adjusting for Mismatched Temporal Resolutions in the Data}

In the joint modeling of neural and behavioral data, the mismatch of the measurement time always occurs due to different temporal resolutions across modalities. In our case, behavioral data (i.e., coordinates of the mouse position) has higher temporal resolution than neural data (i.e., BOLD responses from the MRI machine).

We solve this mismatch problem by imposing partial observations. We assume a latent temporal dynamics kernel $K_{\left(T_{B} \times T_{B}\right)}$, the time vector for the neural measurement $z=\left(z_{1}, \cdots, z_{T_{N}}\right)^{\prime}$, and the time vector for the behavioral measurement $t=\left(t_{1}, \cdots, t_{T_{B}}\right)^{\prime}$ (where $T_{N}$ and $T_{B}$ are the number of samples of neural and behavioral data, respectively). To evaluate the likelihood of the neural data at only a limited observations, we downsize $K$ as follows:

$$
\begin{aligned}
K_{N} & =M^{\prime} K M, \\
M_{\left(T_{B} \times T_{N}\right)} & =[m(i, j)]_{i j} \text { where } m(i, j)=\left\{\begin{array}{l}
1 \text { if } t_{i}=z_{j} \\
0 \text { otherwise }
\end{array}\right.
\end{aligned}
$$

where $i \in\left\{1, \cdots, T_{B}\right\}$ and $j \in\left\{1, \cdots, T_{N}\right\}$. 


\section{Supplementary Information B: Synthetic Data Generation for the Simulation}

In this section we describe the procedures used for generating neural and behavioral data. Table 1 summarizes the parameters used for generating simulated data.

Table 1: Parameters used for generating the simulation data.

\begin{tabular}{lc|lc}
\hline Parameter & Value & Parameter & Value \\
\hline Neural kernel (spatial): Variance & $s_{V}=0.5$ & Observation noise: Neural & $\sigma_{N}^{2}=0.015$ \\
Neural kernel (spatial): Length-scale & $l_{V}=0.75$ & Observation noise: Behavioral & $\sigma_{B}^{2}=0.015$ \\
Neural kernel (latent-temporal): Variance & $s_{X}=1$ & & \\
Neural kernel (latent-temporal): Length-scale & $l_{X}=0.75$ & & \\
Behavioral kernel: Variance & $s_{B}=0.75$ & & \\
Behavioral kernel: Length-scale & $l_{B}=3$ & & \\
\hline
\end{tabular}

\subsection{Latent Dynamics}

The simulated data was defined by pre-defined latent dynamics: given time $t(0 \leq t \leq 100)$, two-dimensional latent dynamics were defined as

$$
\begin{cases}C_{1}(t) & =\sin (t / 8) \\ C_{2}(t) & =\sin (t / 4)\end{cases}
$$

Here we assumed a maximum sampling rate of $1.5 \mathrm{~Hz}$ for generating data (i.e., three measurements every two seconds), producing 151 measurements in total. The latent state matrix $X$ was defined by taking $C_{1}$ and $C_{2}$ as its columns (i.e., $\left.X=\left[C_{1} \mid C_{2}\right]\right)$.

\subsection{Spatial Kernel}

For generating neural data, we assumed measurements from 27 voxels in a three-dimensional grid space. Each dimension could have three points 0,1 , and 2 :

$$
V=\left[\begin{array}{ccc}
0 & 0 & 0 \\
0 & 0 & 1 \\
0 & 0 & 2 \\
0 & 1 & 0 \\
\vdots & \vdots & \vdots \\
2 & 2 & 2
\end{array}\right]
$$

where each row of $V$ specifies the three-dimensional coordinates of a voxel. The spatial kernel $K_{V V}$ was defined as a RBF kernel

$$
K_{V V}=\left[k_{V}\left(v_{i}, v_{j}\right)\right]_{i j}:=\left[s_{V}^{2} \exp \left(-\frac{\left(v_{i}-v_{j}\right)^{2}}{2 l_{V}^{2}}\right)\right]_{i j}
$$

where $i, j \in\{0,1,2\}, v_{i}$ refers to the $i$-th row of $V$, and $s_{V}=0.5$ and $l_{V}=0.75$ are the hyperparameters of the RBF kernel (variance and length-scale, respectively).

\subsection{Data Generation}

For the neural data, we first defined the RBF kernel based on the latent dynamics $X$ :

$$
K_{X X}=\left[k_{X}\left(x_{i}, x_{j}\right)\right]_{i j}:=\left[s_{X}^{2} \exp \left(-\frac{\left(x_{i}-x_{j}\right)^{2}}{2 l_{X}^{2}}\right)\right]_{i j}
$$

where $i, j \in\{1,2, \cdots, 151\}, x_{i}$ refers to the $i$-th row of $X$, and $s_{X}=1$ and $l_{X}=0.75$ are the hyperparameters of the RBF kernel (variance and length-scale, respectively). The kernel $K_{X X}$ was convolved with a double-gamma HRF:

$$
h(t)=\frac{t^{a_{1}-1} b_{1}^{a_{1}} \exp \left(-b_{1} t\right)}{\Gamma\left(a_{1}\right)}-c \frac{t^{a_{2}-1} b_{2}^{a_{2}} \exp \left(-b_{2} t\right)}{\Gamma\left(a_{2}\right)}
$$


where $a_{1}=6, a_{2}=16, b_{1}=b_{2}=1, c=1 / 6, t$ represents time, and $\Gamma(x)=(x-1) !$ is the gamma function. We denote the convolved latent temporal kernel as $K_{X X}^{*}$. (The details of convolving a kernel with an HRF are discussed in "Convolution Processes".)

As discussed in "Adjusting for Mismatched Temporal Resolutions in the Data", one issue in generating neural data is that the temporal resolution of neural measurements do not match that of behavioral measurements (i.e., repetition time $(\mathrm{TR})=2$ seconds, 51 measurements in total). To resolve this mismatch, we assumed partial observation. Given the convolved latent kernel $K_{X X}^{*}$, the time vector for the neural measurement $z=\left(z_{1}, \cdots, z_{51}\right)^{\prime}$, and the time vector for the behavioral measurement $t=\left(t_{1}, \cdots, t_{151}\right)^{\prime}$, we downsized $K_{X X}^{*}$ :

$$
\begin{aligned}
K_{X X(N)} & =M^{\prime} K_{X X}^{*} M, \\
M_{(151 \times 51)} & =[m(i, j)]_{i j} \text { where } m(i, j)=\left\{\begin{array}{l}
1 \text { if } t_{i}=z_{j} \\
0 \text { otherwise }
\end{array}\right.
\end{aligned}
$$

where $i \in\{1, \cdots, 151\}$ and $j \in\{1, \cdots, 51\}$.

Finally, we sampled neural data with a Kronecker-product spatiotemporal kernel defined as $K_{V V} \otimes K_{X X(N)}$ and Gaussian observation noise $\sigma_{N}^{2}=0.015$ :

$$
\operatorname{vec}\left(Y_{N}\right) \sim \mathcal{N}\left(\mathbf{0}, K_{V V} \otimes K_{X X(N)}+\sigma_{N}^{2} I\right)
$$

where $\operatorname{vec}(A)$ represents the vectorization operator that stacks up all the column vectors of $A$. After sampling, the vector was reshaped into a $(51 \times 27)$ matrix. When fitting the GPJM, we used the detrended data by subtracting the voxel-wise mean from each voxel-wise data.

For the behavioral data, we used a Matérn $1 / 2$ kernel with observation noise $\sigma_{B}^{2}=0.015$ :

$$
\begin{aligned}
Y_{B} & \sim \mathcal{N}\left(\mathbf{0}, K_{X X(B)}+\sigma_{B}^{2} I\right), \\
K_{X X(B)} & =\left[k_{B}\left(x_{i}, x_{j}\right)\right]_{i j}:=\left[s_{B}^{2} \exp \left(-\frac{\left\|x_{i}-x_{j}\right\|}{l_{B}}\right)\right]_{i j}
\end{aligned}
$$

where $\sigma_{B}^{2}$ is the observation noise parameter, $I$ is an identity matrix, and $s_{B}=0.75$ and $l_{B}=3$ are the hyperparameters of the Matérn $1 / 2$ kernel (variance and length-scale, respectively). $\|a\|$ represents the Euclidian norm of a vector $a$ : $\|a\|=\sqrt{\sum_{i}\left(a_{i}\right)^{2}}$. When fitting the GPJM, we detrended the data by subtracting the overall mean from the original values. 


\section{Supplementary Information C: Simulation Results}

\subsection{Estimated Kernel and Noise Parameters}

Table 2 provides the maximum a posteriori estimates of the kernel and noise parameters (rounded up to three decimal points).

Table 2: Simulation: Maximum a posteriori kernel and noise parameters. Rounded up to three decimal places.

\begin{tabular}{lc|lc}
\hline Parameter & Estimated value & Parameter & Estimated value \\
\hline Temporal kernel: Variance & 0.480 & Observation noise: Neural & 0.016 \\
Temporal kernel: Length-scale & 9.216 & Observation noise: Behavioral & 0.003 \\
Neural kernel (Spatial):Variance & 1 (fixed) & \\
Neural kernel (Spatial): Length-scale & 1.235 & \\
Neural kernel (latent-temporal): & 0.042 & \\
Variance & & \\
Neural kernel (latent-temporal): & 0.324 & \\
Length-scale & & \\
Behavioral kernel (latent-temporal): & 0.497 & \\
Variance & & \\
Behavioral kernel (latent-temporal): & 1.624 & \\
Length-scale & & \\
\hline
\end{tabular}

\subsection{Fits to Simulated Data}

Figures 6 and 7 show the model fit to the simulated neural and behavioral data, respectively. Black lines present the simulated data. Red solid lines show the mean predictions from the model, and red dotted lines represent the $95 \%$ predictive intervals. 


\section{Supplementary Information D: fMRI Experiment}

\subsection{MRI Data Acquisition and Preprocessing}

MRI data was collected on a Siemens 3T MAGNETOM Prisma MRI system with a 32 channel head coil at the Center for Cognitive and Behavioral Brain Imaging at The Ohio State University. Structural images were obtained with an axial T1-weighted sequence $(\mathrm{TR}=1900 \mathrm{~ms}$, TE $=4.44 \mathrm{~ms}, \mathrm{TI}=991 \mathrm{~ms}$, flip angle $=8$ degrees, field of view $=256$ x $256 \mathrm{~mm}, 176$ slices, voxel size $=1 \times 1 \times 1 \mathrm{~mm}$ ). During each block of the task, we used an axial multiband-EPI sequence $(\mathrm{TR}=1000 \mathrm{~ms}, \mathrm{TE}=28 \mathrm{~ms}$, flip angle $=60$ degrees, field of view $=216 \times 216 \mathrm{~mm}, 45$ interleaved slices with no spacing, voxel size $=3 \times 3 \times 3 \mathrm{~mm}$, multiband acceleration factor $=3,784$ volumes) to acquire whole-brain BOLD signal data. Lastly, we acquired a field map $(\mathrm{TR}=512 \mathrm{~ms}$, TE1 $=5.19 \mathrm{~ms}$, TE2 $=7.65 \mathrm{~ms}$, flip angle $=60 \mathrm{degrees}$, field of view $=225 \times 225 \mathrm{~mm}, 45$ slices, voxel size $=2 \times 2 \times 3 \mathrm{~mm}$ ) to correct for static field inhomogeneity during preprocessing.

FMRI data preprocessing and analysis was performed primarily with FEAT (FMRI Expert Analysis Tool; [44]) Version 6.00, a tool within FSL (FMRIB's Software Library; https://fsl.fmrib.ox.ac.uk/fsl/). Preprocessing for each block of the 4D task data consisted of head motion correction using MCFLIRT [46], fieldmap-based EPI distortion correction, removal of non-brain structures with BET [47], spatial smoothing with a 4.5 mm FWHM Gaussian kernel, highpass filtering with a 100 second cutoff, and normalization via grand-mean scaling. The EPI data was first registered to the T1-weighted image using the brain-boundary-based registration method in FLIRT [46, 48], then transformed into standard space using the same transformation matrix created when registering the T1-weighted image to standard space (MNI152 with $1 \mathrm{~mm}$ spatial resolution) with FNIRT. The T1-weighted image was segmented into gray matter, white matter, and cerebrospinal fluid using FAST [49] then warped into EPI space using the aforementioned transformation matrices. The transformed WM and CSF masks were then applied to the EPI data and a mean time series was extracted for each.

\subsection{Regions of Interest}

The resulting clusters from the fixed-effects analysis were corrected for family-wise error at $p<.05$; however, they contained significant overlap. Specifically, many of the clusters resulting from the contrasts comparing non-zero coherence to participant response or zero-coherence shared significant overlap with clusters from the main predictors (participant responses, non-zero stimulus coherence, and zero stimulus coherence). In an effort to eliminate redundant signal in the neural data, we selected clusters that did not overlap. Enforcing this constraint yielded 16 ROIs (7 associated with non-zero coherence, 9 associated with participant response). Figure 8 shows the $Z$-statistic map for these ROIs from the fixed-effects analysis (see Tables 3 and 4 for details). Individual masks of these ROIs were generated then warped into the preprocessed functional data space from the first block of the task. Finally, the masks were applied to the functional data and a mean time series vector was extracted for each ROI.

Table 3: Regions of interest associated with non-zero coherence. Data presented in $1 \mathrm{~mm}$ MNI space.

\begin{tabular}{lccccc}
\hline Region & X & Y & Z & Cluster size & Max Z score \\
\hline R visual cortex V1 & 7 & -72 & -9 & 3387 & 3.93 \\
R inferior parietal lobule & 49 & -40 & 9 & 1903 & 4.48 \\
L visual cortex V2 & -9 & -88 & 26 & 1543 & 4.11 \\
L frontal pole & -48 & 48 & -14 & 1487 & 3.34 \\
R crus II & 29 & -69 & -41 & 1266 & 3.46 \\
R premotor cortex & 32 & -23 & 64 & 1159 & 3.82 \\
L inferior frontal gyrus & -54 & 23 & 0 & 1003 & 3.07 \\
\hline
\end{tabular}

\subsection{Fits to Real Data}

Tables 5 and 6 show the maximum a posteriori estimates of the kernel and observation noise parameters from the fMRI experiment. Table 5 reports the results from the GPJM assuming three-dimensional latent dynamics. Table 6 reports the results from the GPJM assuming four-dimensional latent dynamics.

Figures 9, 10, 11 and 12 show the model fit to the neural and behavioral data observed from the fMRI experiment. The first two figures are the results from the Gaussian process joint model with three-dimensional latent dynamics. The latter two figures are the results from the Gaussian process joint model with four-dimensional latent dynamics. Black lines present the observations. Red solid lines show the mean predictions from the model, and orange dotted lines represent the $95 \%$ predictive intervals. 
A PREPRINT - NOVEMBER 13, 2019

Table 4: Regions of interest associated with participant response. Data presented in $1 \mathrm{~mm}$ MNI space.

\begin{tabular}{lccccc}
\hline Region & X & Y & Z & Cluster size & Max Z score \\
\hline L superior parietal lobule & -10 & -56 & 68 & 8664 & 6.71 \\
L primary auditory cortex & -53 & -21 & 10 & 5151 & 4.09 \\
R inferior parietal lobule & 60 & -25 & 13 & 3923 & 4.71 \\
L primary motor cortex & -52 & 0 & 32 & 2522 & 3.65 \\
L premotor cortex & -44 & -3 & 61 & 1606 & 4.24 \\
R premotor cortex & 10 & -6 & 72 & 1579 & 3.78 \\
L inferior parietal lobule & -44 & -64 & 11 & 1366 & 4.27 \\
R primary auditory cortex & 54 & -14 & 6 & 1311 & 3.90 \\
R primary somatosensory cortex & 66 & -3 & 22 & 1066 & 3.40 \\
\hline
\end{tabular}

Table 5: fMRI experiment: Maximum a posteriori kernel and noise parameters of the Gaussian process joint model with three-dimensional latent dynamics. Rounded up to three decimal places. fMRI: functional magnetic resonance imaging.

\begin{tabular}{lc|ll}
\hline & Value & & Value \\
\hline Temporal kernel: Variance & 0.014 & Observation noise: Neural & 0.039 \\
Temporal kernel: Length-scale & 0.657 & Observation noise: Behavioral & 0.105 \\
Neural kernel: Variance & 17.137 & & \\
Neural kernel: Length-scale & {$[0.186,0.227,0.525]$} & & \\
Behavioral kernel: Variance & 14.308 & & \\
Behavioral kernel: Length-scale & {$[0.578,0.546,0.399]$} & & \\
\hline
\end{tabular}

Figures 13 and 14 present the latent dynamics estimated from the four-dimensional GPJM. Figure 13 shows the timeseries representations of the dynamics, whereas Figure 14 provides two three-dimensional trajectory representations of the latent dynamics. For visual clarity, we smoothed the dynamics used in Figure 14]using the Savitzky-Golay filter [50] with a window size of 51 and the third-order polynomial approximation.

\subsection{Model Evaluation}

We used log posterior predictive density (PPD) and root mean squared error (RMSE) for evaluating the predictive performance of each model variant. First, the log PPD was computed using the posterior predictive mean and covariance functions for the test data.

We separate the training behavioral data $\mathbf{y}$, the test behavioral data $\mathbf{y}^{*}$, part of the latent dynamics $X$ trained by both neural and training behavioral data, and another part of the latent dynamics $X^{*}$ trained only by the neural data. The latent dynamics the predictive mean $\overline{\mathbf{f}}^{*}$ and predictive covariance matrix $\Sigma^{*}$ are analytically derived as follows [17]:

$$
\begin{aligned}
\overline{\mathbf{f}}^{*}= & K\left(X^{*}, X\right)\left[K(X, X)+\sigma_{\epsilon}^{2} I\right]^{-1} \mathbf{y} \\
\Sigma^{*}= & K\left(X^{*}, X^{*}\right) \\
& -K\left(X^{*}, X\right)\left[K(X, X)+\sigma_{\epsilon}^{2} I\right]^{-1} K\left(X, X^{*}\right)
\end{aligned}
$$

where $K(M, N)=\left[k\left(m_{i}, n_{j}\right)\right]_{i j}$ is the kernel whose elements are determined by the $i$-th row of matrix $M$ (denoted $m_{i}$ ) and the $j$-th row of matrix $N$ (denoted $n_{j}$ ).

We derived the posterior predictive mean and variance with the equations above using the trained latent dynamics, complete neural data, and partially observed behavioral data. Then, the $\log$ PPD for the test behavioral data $\mathbf{y}^{*}$ is

$$
\log P P D=-\frac{1}{2}\left[\log \operatorname{det} \Sigma^{*}+\left(\mathbf{y}^{*}-\overline{\mathbf{f}}^{*}\right)^{\prime} \Sigma^{*}\left(\mathbf{y}^{*}-\overline{\mathbf{f}}^{*}\right)+k \log 2 \pi\right]
$$

where $\operatorname{det} M$ means the determinant of a matrix $M$, and $k$ is the number of test data points. The RMSE was defined as

$$
R M S E=\sqrt{\left(\mathbf{y}^{*}-\overline{\mathbf{f}}^{*}\right)^{\prime}\left(\mathbf{y}^{*}-\overline{\mathbf{f}}^{*}\right) / k} .
$$

High PPD and low RMSE suggest that a model provides better predictions about the unobserved data. 
Table 6: fMRI experiment: Maximum a posteriori kernel and noise parameters of the Gaussian process joint model with four-dimensional latent dynamics. Rounded up to three decimal places. fMRI: functional magnetic resonance imaging.

\begin{tabular}{lc|cc}
\hline & Value & & Value \\
\hline Temporal kernel: Variance & 0.010 & Observation noise: Neural & 0.038 \\
Temporal kernel: Length-scale & 0.597 & Observation noise: Behavioral & 0.001 \\
Neural kernel: Variance & 22.039 & & \\
Neural kernel: Length-scale & {$[0.440,0.260,0.202,21.362]$} & \\
Behavioral kernel: Variance & 8.649 & \\
Behavioral kernel: Length-scale & {$[3.225,1.352,4.773,0.571]$} & & \\
\hline
\end{tabular}

\section{References}

[1] Brandon M Turner, Birte U Forstmann, and Mark Steyvers. Joint models of neural and behavioral data. Springer, New York, NY, 2019.

[2] Giles Skey Brindley. Physiology of the retina and the visual pathway. Williams and Wilkins, Oxford, England, Second edition, 1970.

[3] Davida Y Teller. Linking propositions. Vision Research, 24:1233-1246, 1984.

[4] Jeffrey D Schall. On Building a Bridge Between Brain and Behavior. Annual Review of Psychology, 55:23-50, 2004.

[5] Brandon M Turner, James J Palestro, Steven Miletić, and Birte U Forstmann. Advances in techniques for imposing reciprocity in brain-behavior relations. Neuroscience \& Biobehavioral Reviews, 102:327-336, 2019.

[6] Birte U Forstmann, Gilles Dutilh, Scott Brown, Jane Neumann, D Yves Von Cramon, K Richard Ridderinkhof, and Eric-Jan Wagenmakers. Striatum and pre-SMA facilitate decision-making under time pressure. Proceedings of the National Academy of Sciences, 105(45):17538-17542, 2008.

[7] Birte U Forstmann, Alfred Anwander, Andreas Schäfer, Jane Neumann, Scott Brown, Eric-Jan Wagenmakers, Rafal Bogacz, and Robert Turner. Cortico-striatal connections predict control over speed and accuracy in perceptual decision making. Proceedings of the National Academy of Sciences, 107(36):15916-15920, 2010.

[8] Birte U Forstmann, Eric-Jan Wagenmakers, Tom Eichele, Scott Brown, and John T Serences. Reciprocal relations between cognitive neuroscience and formal cognitive models: Opposites attract? Trends in Cognitive Sciences, 15(6):272-279, 2011.

[9] Brandon M Turner, Birte U Forstmann, Bradley C Love, Thomas J Palmeri, and Leendert Van Maanen. Approaches to analysis in model-based cognitive neuroscience. Journal of Mathematical Psychology, 76:65-79, 2017.

[10] Brandon M Turner, Birte U Forstmann, Eric-Jan Wagenmakers, Scott D Brown, Per B Sederberg, and Mark Steyvers. A Bayesian framework for simultaneously modeling neural and behavioral data. NeuroImage, 72:193206, 2013.

[11] Brandon M Turner, Per B Sederberg, Scott D Brown, and Mark Steyvers. A method for efficiently sampling from distributions with correlated dimensions. Psychological Methods, 18:368-384, 2013.

[12] Brandon M Turner, Christian A Rodriguez, Tony M Norcia, Samuel M McClure, and Mark Steyvers. Why more is better: A method for simultaneously modeling EEG, fMRI, and Behavior. NeuroImage, 128:96-115, 2016.

[13] Brandon M Turner, Leendert Van Maanen, and Birte U Forstmann. Combining Cognitive Abstractions with Neurophysiology: The Neural Drift Diffusion Model. Psychological Review, 122:312-336, 2015.

[14] Brandon M Turner, Ting Wang, and E Merkel. Factor analysis linking functions for simultaneously modeling neural and behavioral data. NeuroImage, 153:28-48, 2017.

[15] James J Palestro, Giwon Bahg, Per B Sederberg, Zhong-Lin Lu, Mark Steyvers, and Brandon M Turner. A tutorial on joint models of neural and behavioral measures of cognition. Journal of Mathematical Psychology, 84:20-48, 2018.

[16] James M Shine, Michael Breakspear, Peter T Bell, Kaylena A Ehgoetz Martens, Richard Shine, Oluwasanmi Koyejo, Olaf Sporns, and Russell A Poldrack. Human cognition involves the dynamic integration of neural activity and neuromodulatory systems. Nature Neuroscience, 22:289-296, 2019.

[17] Christopher K I Williams and Carl Edward Rasmussen. Gaussian processes for machine learning. MIT Press, Cambridge, MA, 2006. 
[18] Eric Schulz, Maarten Speekenbrink, and Andreas Krause. A tutorial on Gaussian process regression: Modelling, exploring, and exploiting functions. Journal of Mathematical Psychology, 85:1-16, 2018.

[19] Radford M Neal. Bayesian Learning for Neural Networks. Springer, New York, NY, 1996.

[20] M. Shvartsman, N. Sundaram, M. C. Aoi, A. Charles, T. C. Wilke, and J. D. Cohen. "Matrix-normal models for fMRI analysis". arXiv, arXiv:1711.03058.

[21] Neil D Lawrence. Gaussian process latent variable models for visualisation of high dimensional data. In Advances in Neural Information Processing Systems, pages 329-336, 2004.

[22] Neil D Lawrence. Probabilistic non-linear principal component analysis with Gaussian process latent variable models. Journal of Machine Learning Research, 6:1783-1816, 2005.

[23] Neil Lawrence. Hierarchical Gaussian process latent variable models. In G. Ghahramani, editor, Proceedings of the International Conference in Machine Learning, pages 481-488, New York, NY, 2007. ACM.

[24] Michalis Titsias and Neil D Lawrence. Bayesian Gaussian process latent variable model. In Proceedings of the Thirteenth International Conference on Artificial Intelligence and Statistics, pages 844-851, 2010.

[25] Anqi Wu, Nicholas A Roy, Stephen Keeley, and Jonathan W Pillow. Gaussian process based nonlinear latent structure discovery in multivariate spike train data. In Advances in Neural Information Processing Systems, pages 3496-3505, 2017.

[26] Geoffrey M Boynton, Jonathan B Demb, Gary H Glover, and David J Heeger. Neuronal basis of contrast discrimination. Vision research, 39(2):257-269, 1999.

[27] Jesse Rissman, Adam Gazzaley, and Mark D'Esposito. Measuring functional connectivity during distinct stages of a cognitive task. NeuroImage, 23:752-763, 2004.

[28] Dave Higdon. Space and space-time modeling using process convolutions. In Quantitative methods for current environmental issues, pages 37-56. Springer-Verlag, London, 2002.

[29] Mauricio Álvarez and Neil D Lawrence. Sparse convolved Gaussian processes for multi-output regression. In Advances in Neural Information Processing Systems, pages 57-64, 2009.

[30] Mauricio A Álvarez and Neil D Lawrence. Computationally efficient convolved multiple output Gaussian processes. Journal of Machine Learning Research, 12:1459-1500, 2011.

[31] Gary H Glover. Deconvolution of impulse response in event-related bold fmri1. NeuroImage, 9:416-429, 1999.

[32] Edwin V Bonilla, Kian M Chai, and Christopher Williams. Multi-task Gaussian process prediction. In Advances in Neural Information Processing Systems, pages 153-160, 2008.

[33] Seth Flaxman, Andrew Wilson, Daniel Neill, Hannes Nickisch, and Alex Smola. Fast Kronecker inference in Gaussian processes with non-Gaussian likelihoods. In International Conference on Machine Learning, pages 607-616, 2015.

[34] Tilmann Gneiting. Nonseparable, stationary covariance functions for space-time data. Journal of the American Statistical Association, 97:590-600, 2002.

[35] Birte U Forstmann and Eric-Jan Wagenmakers. Model-based cognitive neuroscience: A conceptual introduction. In An introduction to model-based cognitive neuroscience, pages 139-156. Springer, 2015.

[36] B. M. Turner. Constraining cognitive abstractions through Bayesian modeling. In B. U. Forstmann and E.-J. Wagenmakers, editors, An introduction to model-based cognitive neuroscience, pages 199-220. Springer, New York, 2015.

[37] Sumon Ahmed, Magnus Rattray, and Alexis Boukouvalas. GrandPrix: scaling up the Bayesian GPLVM for single-cell data. Bioinformatics, 35(1):47-54, 2018.

[38] Matthew Galdo, Giwon Bahg, and Brandon Turner. Variational Bayes methods for cognitive science. Psychological Methods, 2019. Advance online publication. http://dx.doi.org/10.1037/met0000242.

[39] Markus Heinonen, Henrik Mannerström, Juho Rousu, Samuel Kaski, and Harri Lähdesmäki. Non-stationary Gaussian process regression with Hamiltonian monte carlo. In Proceedings of Machine Learning Research 51: Artificial Intelligence and Statistics, pages 732-740, 2016.

[40] De G Matthews, G Alexander, Mark Van Der Wilk, Tom Nickson, Keisuke Fujii, Alexis Boukouvalas, Pablo León-Villagrá, Zoubin Ghahramani, and James Hensman. Gpflow: A gaussian process library using TensorFlow. The Journal of Machine Learning Research, 18:1299-1304, 2017.

[41] Martín Abadi, Paul Barham, Jianmin Chen, Zhifeng Chen, Andy Davis, Jeffrey Dean, Matthieu Devin, Sanjay Ghemawat, Geoffrey Irving, Michael Isard, et al. Tensorflow: A system for large-scale machine learning. In The 12th USENIX Symposium on Operating Systems Design and Implementation, pages 265-283, 2016. 
[42] Ciyou Zhu, Richard H Byrd, Peihuang Lu, and Jorge Nocedal. Algorithm 778: L-bfgs-b: Fortran subroutines for large-scale bound-constrained optimization. ACM Transactions on Mathematical Software, 23:550-560, 1997.

[43] Diederik P Kingma and Jimmy Ba. Adam: A method for stochastic optimization. arXiv preprint arXiv:1412.6980, 2014.

[44] Mark W Woolrich, Saad Jbabdi, Brian Patenaude, Michael Chappell, Salima Makni, Timothy Behrens, Christian Beckmann, Mark Jenkinson, and Stephen M Smith. Bayesian analysis of neuroimaging data in FSL. NeuroImage, 45:S173-S186, 2009.

[45] Mark W Woolrich, Brian D Ripley, Michael Brady, and Stephen M Smith. Temporal autocorrelation in univariate linear modeling of FMRI data. NeuroImage, 14(6):1370-1386, 2001.

[46] Mark Jenkinson, Peter Bannister, Michael Brady, and Stephen Smith. Improved optimization for the robust and accurate linear registration and motion correction of brain images. NeuroImage, 17(2):825-841, 2002.

[47] Stephen M Smith. Fast robust automated brain extraction. Human Brain Mapping, 17(3):143-155, 2002.

[48] Douglas N Greve and Bruce Fischl. Accurate and robust brain image alignment using boundary-based registration. NeuroImage, 48(1):63-72, 2009.

[49] Yongyue Zhang, Michael Brady, and Stephen Smith. Segmentation of brain MR images through a hidden markov random field model and the expectation-maximization algorithm. IEEE Transactions on Medical Imaging, 20(1):45-57, 2001.

[50] Abraham Savitzky and Marcel J E Golay. Smoothing and differentiation of data by simplified least squares procedures. Analytical Chemistry, 36:1627-1639, 1964. 

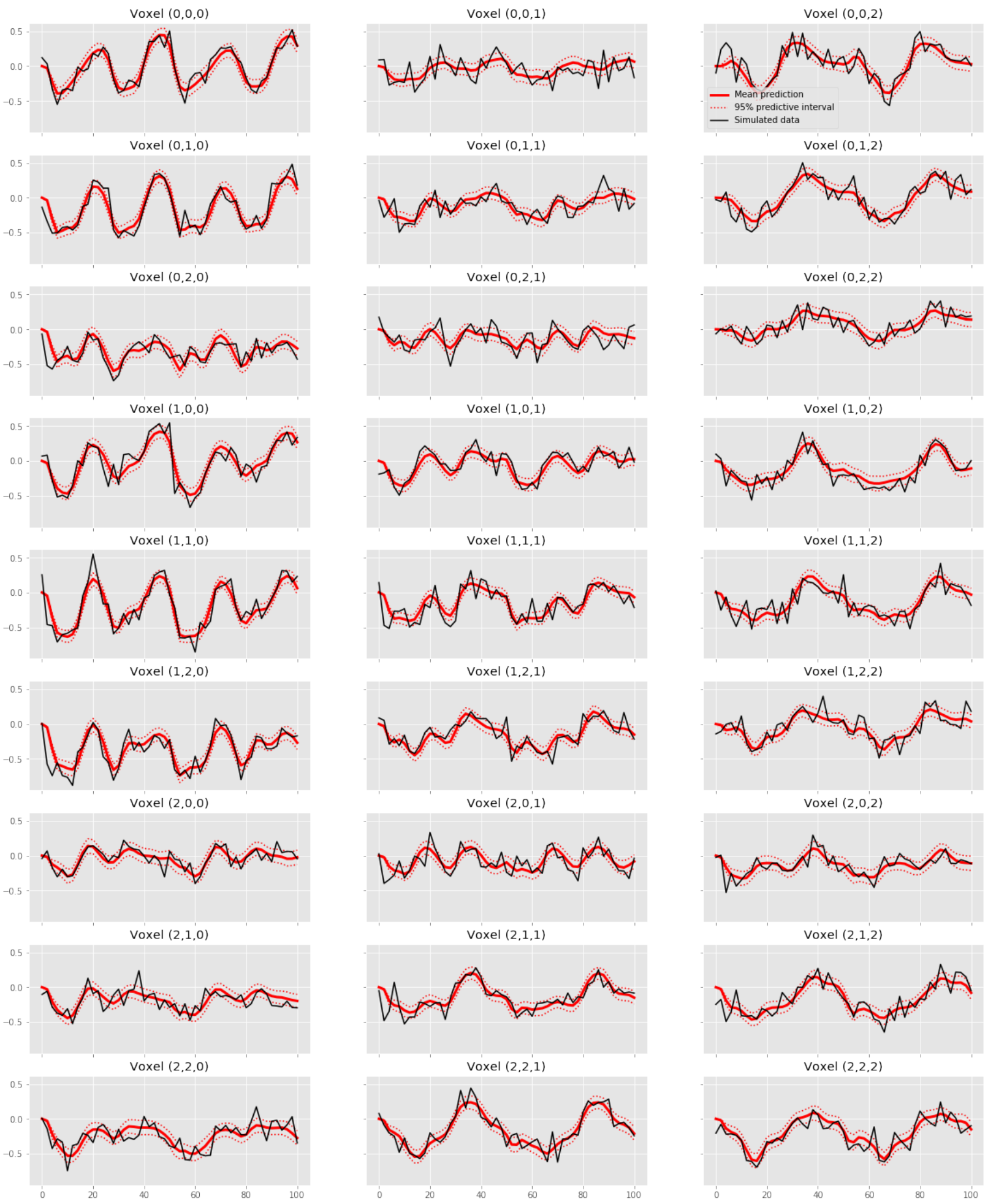

Voxel $(2,1,2)$

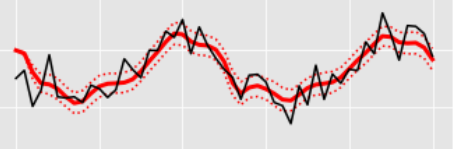

Voxel $(2,2,2)$

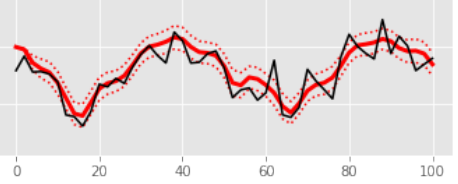

Figure 6: Model fits to the simulated BOLD responses. Black and red solid lines are the simulated data and the mean predictions from the model, respectively. Red dotted lines represent the $95 \%$ predictive intervals of the model prediction. BOLD: blood oxygenation level dependent. 


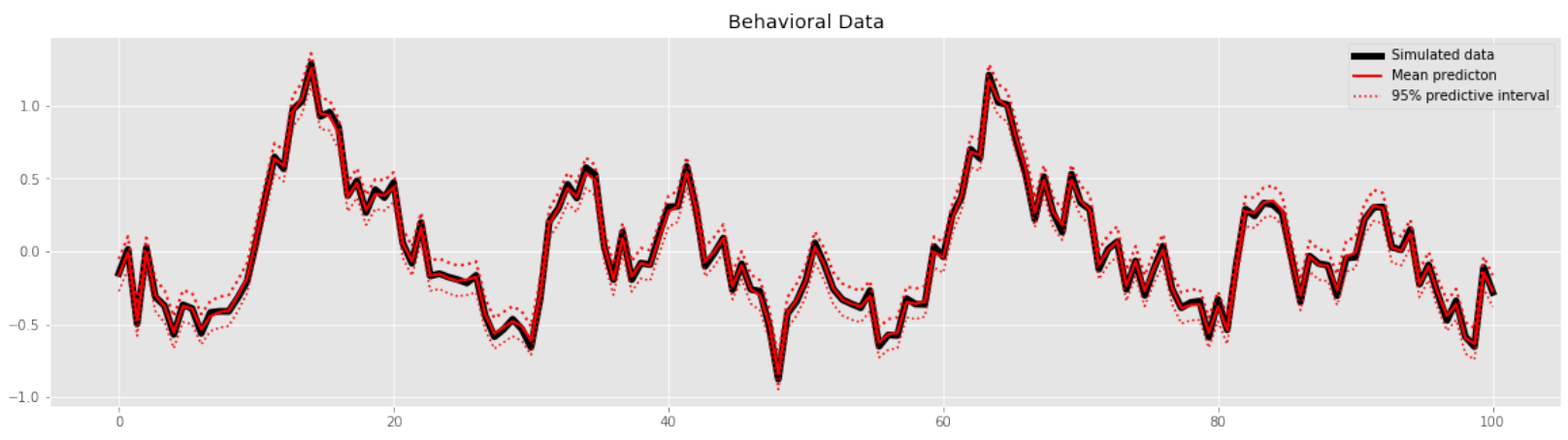

Figure 7: Model fits to the simulated behavioral data. Black and red solid lines are the simulated data and the mean predictions from the model, respectively. Red dotted lines represent the $95 \%$ predictive intervals of the model prediction.

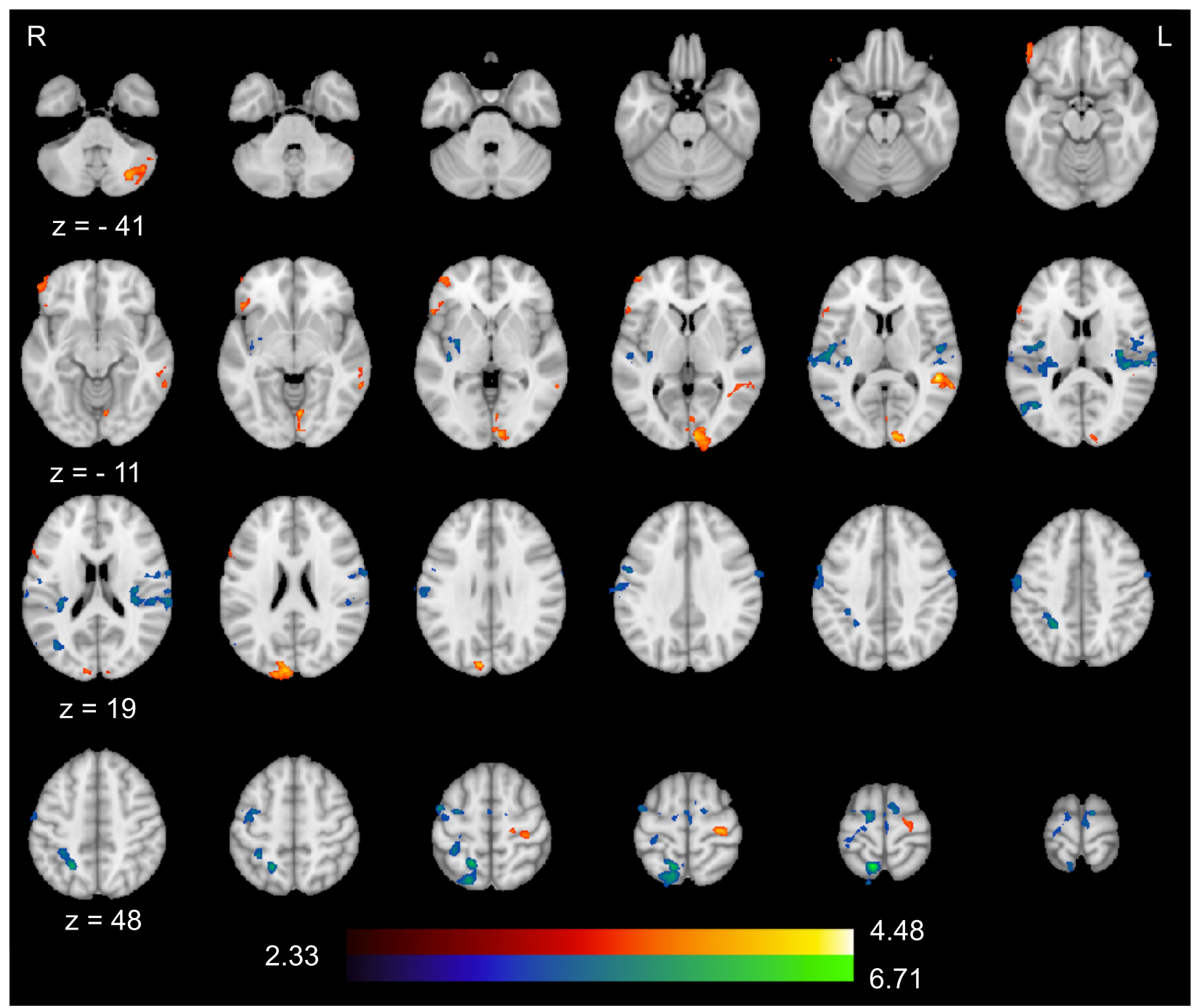

Figure 8: Map of $Z$ statistics from regions of interest associated with non-zero coherence (red-yellow) and participant response (blue-green) resulting from the fixed-effects analysis. Clusters were thresholded at $Z \geq 2.33$ and corrected for family-wise error at $p<.05$. 


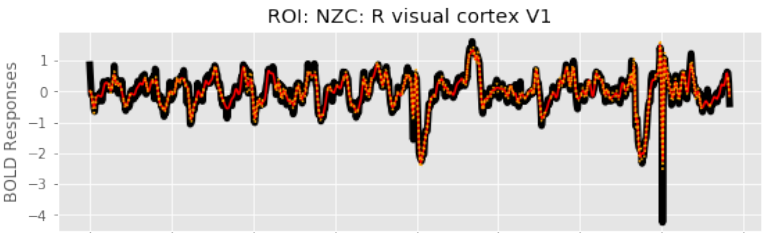

ROI: NZC: L visual cortex V2

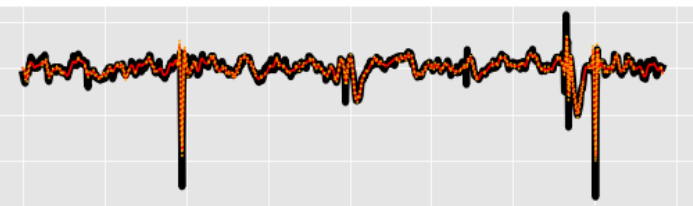

ROI: NZC: R crus II

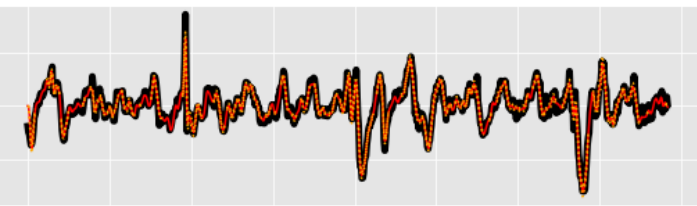

ROI: NZC: L inferior frontal gyrus
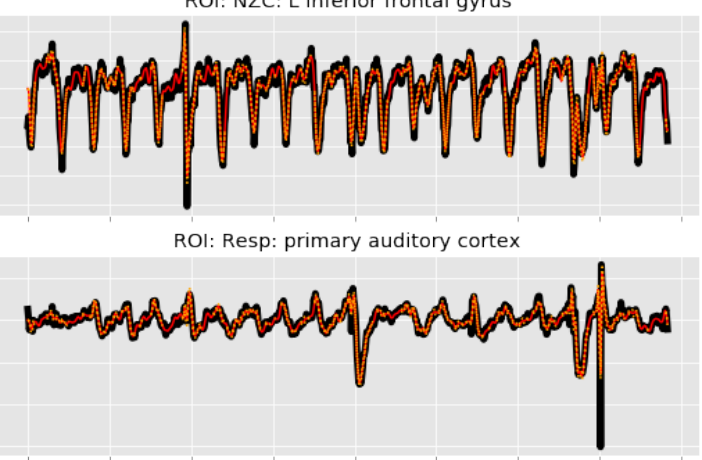

ROI: Resp: L primary motor cortex

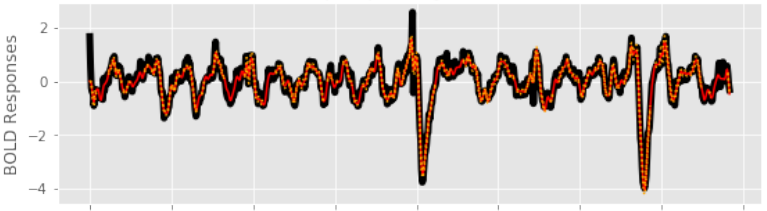

ROI: Resp: R premotor cortex

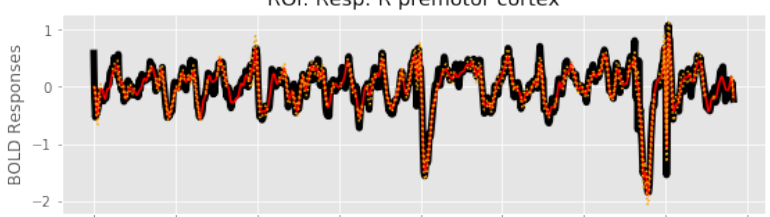

ROI: Resp: R primary auditory cortex

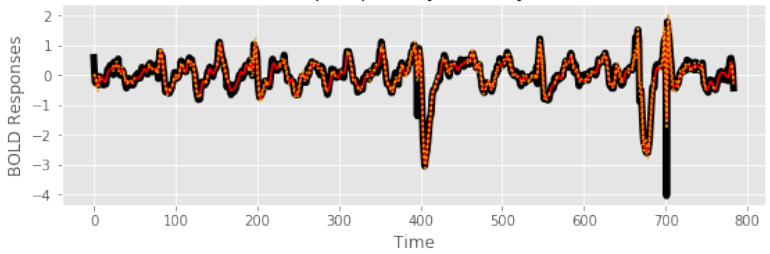

ROI: NZC: R inferior parietal lobule

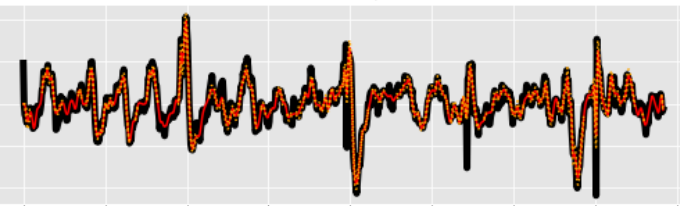

ROI: NZC: L frontal pole

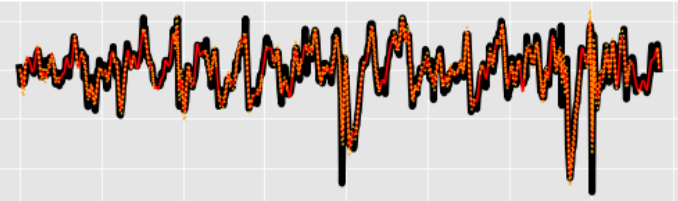

ROI: NZC: R premotor cortex

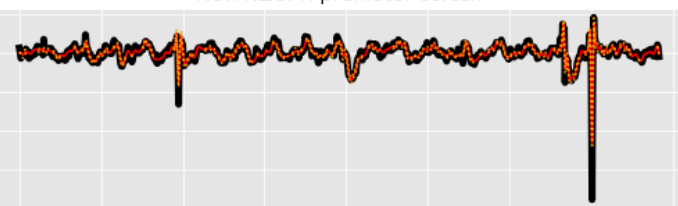

ROI: Resp: L superior parietal lobule

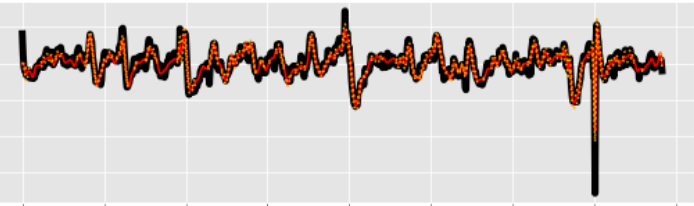

ROI: Resp: R inferior patrietal lobule

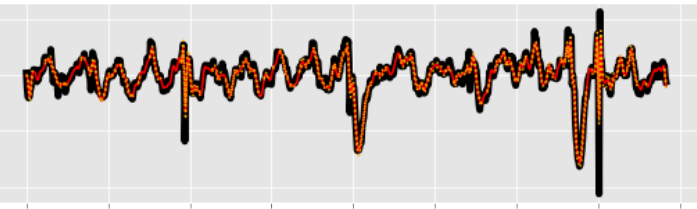

ROI: Resp: L premotor cortex

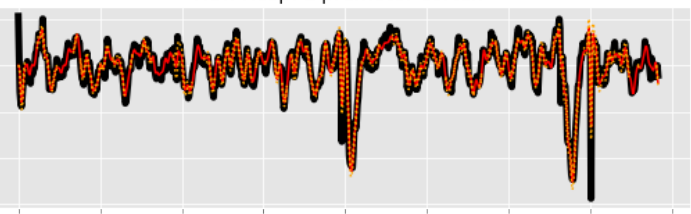

ROI: Resp: L inferior parietal lobule

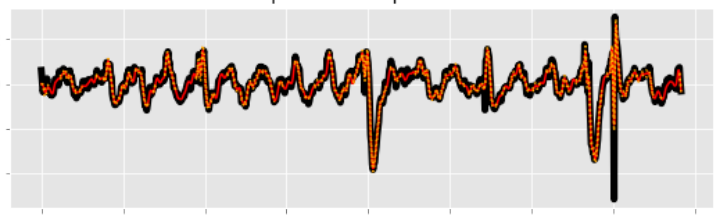

ROI: R primary somatosensory cortex

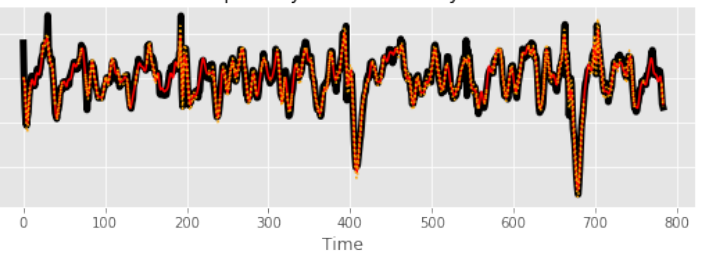

Figure 9: Fits of the GPJM with three-dimensional latent dynamics to the BOLD responses observed from the fMRI experiment. Black and red solid lines are the observed data and the mean predictions from the model, respectively. Orange dotted lines represent the $95 \%$ predictive intervals of the model prediction. ROI: Regions of interest. NZC: Regions of interest associated with non-zero coherence (see Table 3). Resp: Regions of interest with participant response (see Table 47. GPJM: Gaussian process joint model. BOLD: blood oxygenation level dependent. fMRI: functional magnetic resonance imaging. 
Behavioral Data

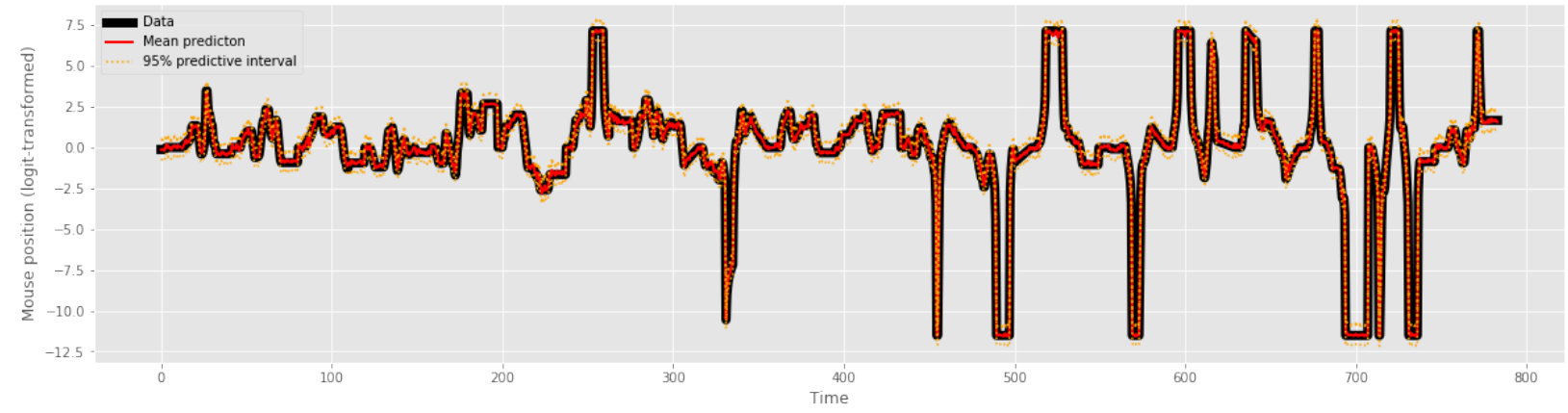

Figure 10: Fits of the GPJM with three-dimensional latent dynamics to the behavioral data observed from the fMRI experiment. Black and red solid lines are the observed data and the mean predictions from the model, respectively. Orange dotted lines represent the $95 \%$ predictive intervals of the model prediction. GPJM: Gaussian process joint model. fMRI: functional magnetic resonance imaging. 


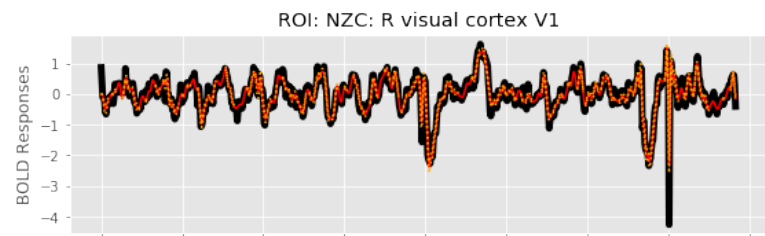

ROI: NZC: L visual cortex V2

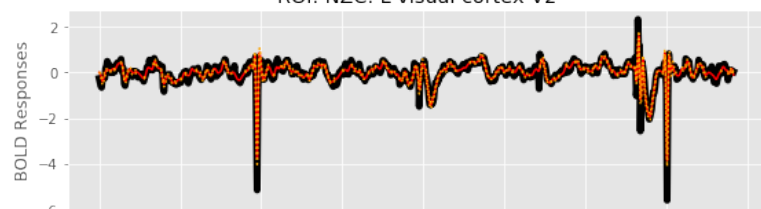

ROI: NZC: R crus II

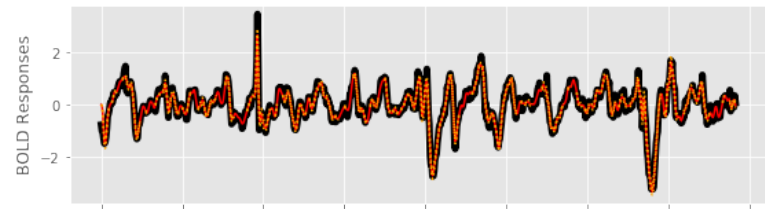

ROI: NZC: L inferior frontal gyrus

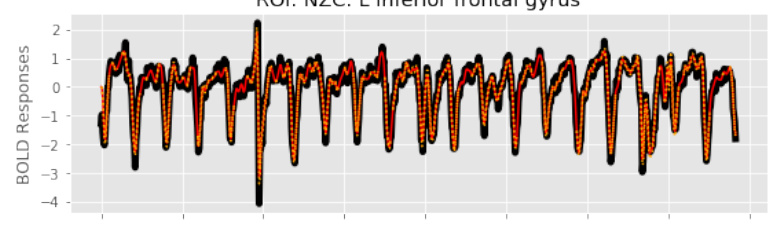

ROI: Resp: primary auditory cortex

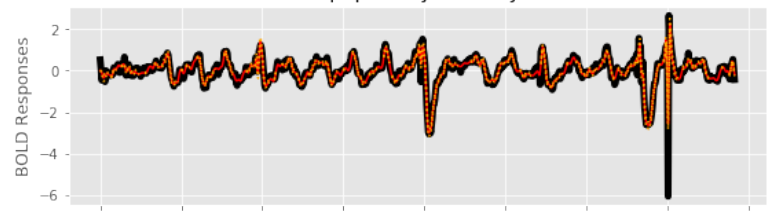

ROI: Resp: L primary motor cortex

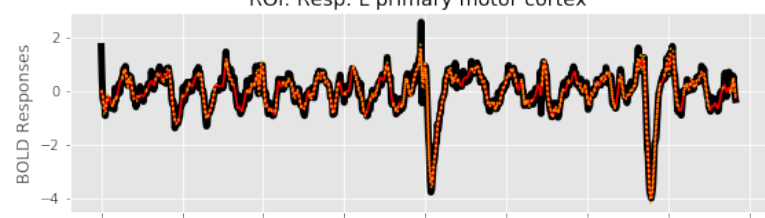

ROI: Resp: R premotor cortex

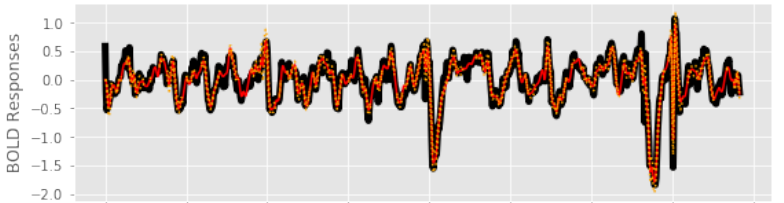

ROI: Resp: R primary auditory cortex

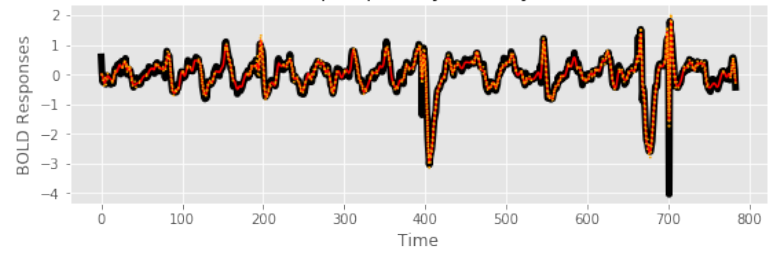

ROI: NZC: R inferior parietal lobule

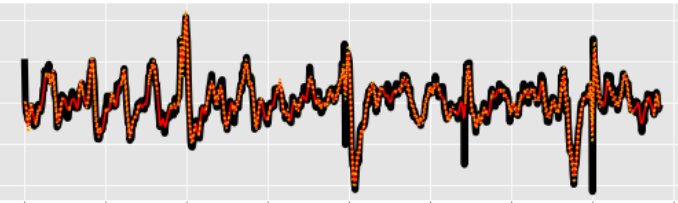

ROI: NZC: L frontal pole

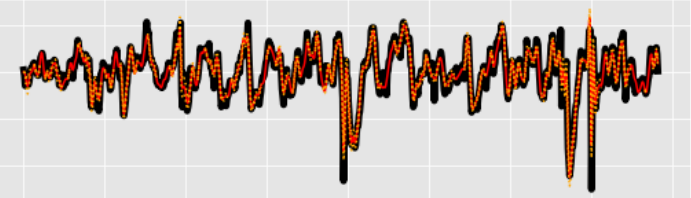

ROI: NZC: R premotor cortex

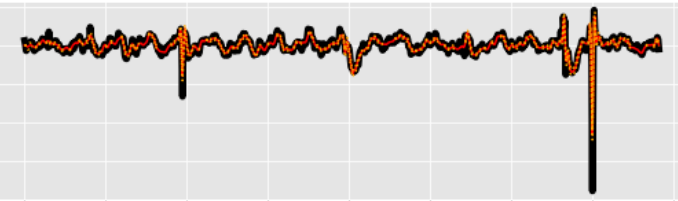

ROI: Resp: L superior parietal lobule

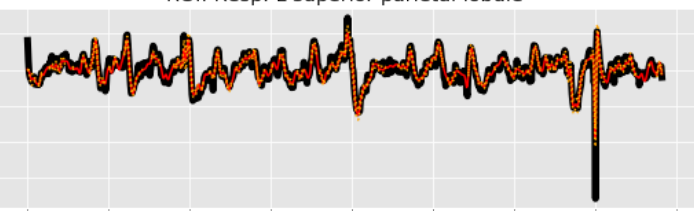

ROI: Resp: R inferior patrietal lobule

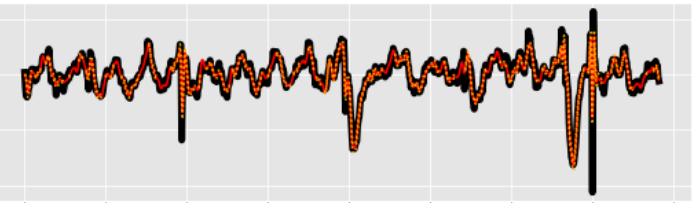

ROI: Resp: L premotor cortex

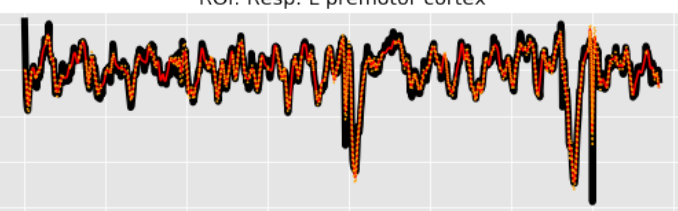

ROI: Resp: L inferior parietal lobule

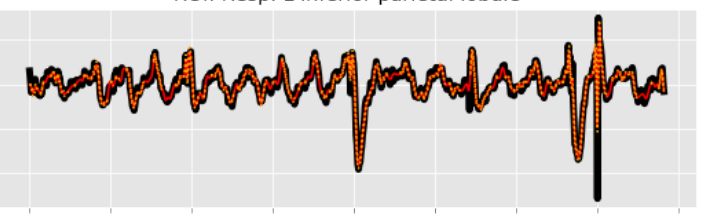

ROI: R primary somatosensory cortex

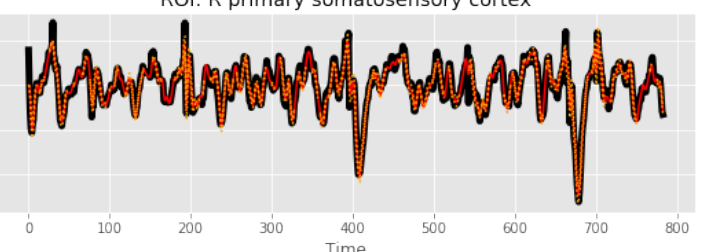

Figure 11: Fits of the GPJM with four-dimensional latent dynamics to the BOLD responses observed from the fMRI experiment. Black and red solid lines are the observed data and the mean predictions from the model, respectively. Orange dotted lines represent the $95 \%$ predictive intervals of the model prediction. ROI: Regions of interest. NZC: Regions of interest associated with non-zero coherence (see Table 3). Resp: Regions of interest with participant response (see Table 4). GPJM: Gaussian process joint model. fMRI: functional magnetic resonance imaging. 


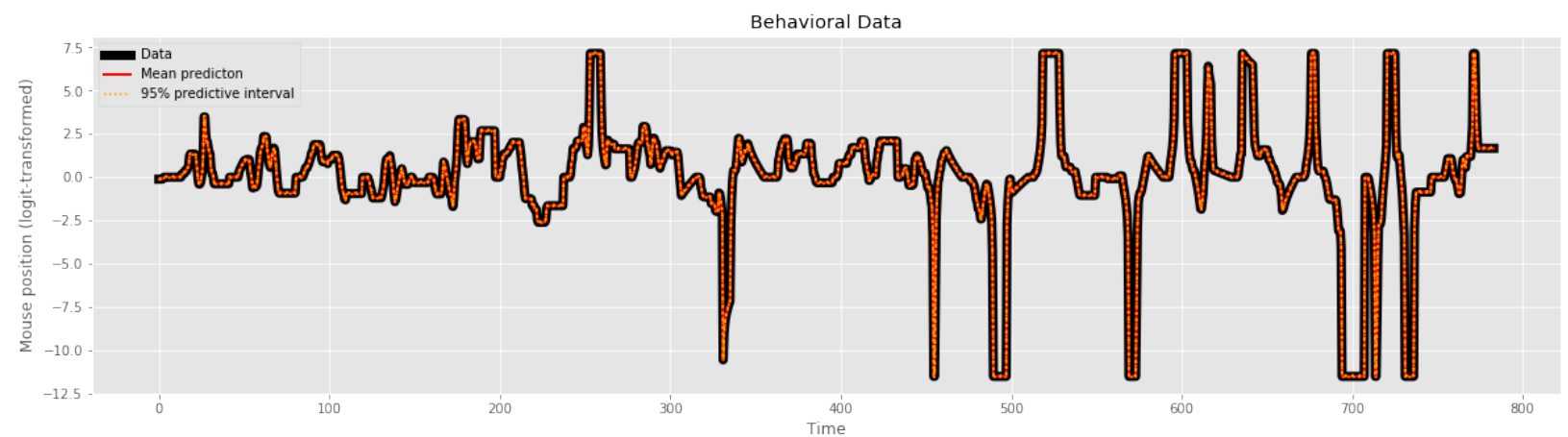

Figure 12: Fits of the GPJM with four-dimensional latent dynamics to the behavioral data observed from the fMRI experiment. Black and red solid lines are the observed data and the mean predictions from the model, respectively. Orange dotted lines represent the $95 \%$ predictive intervals of the model prediction. GPJM: Gaussian process joint model. fMRI: functional magnetic resonance imaging.

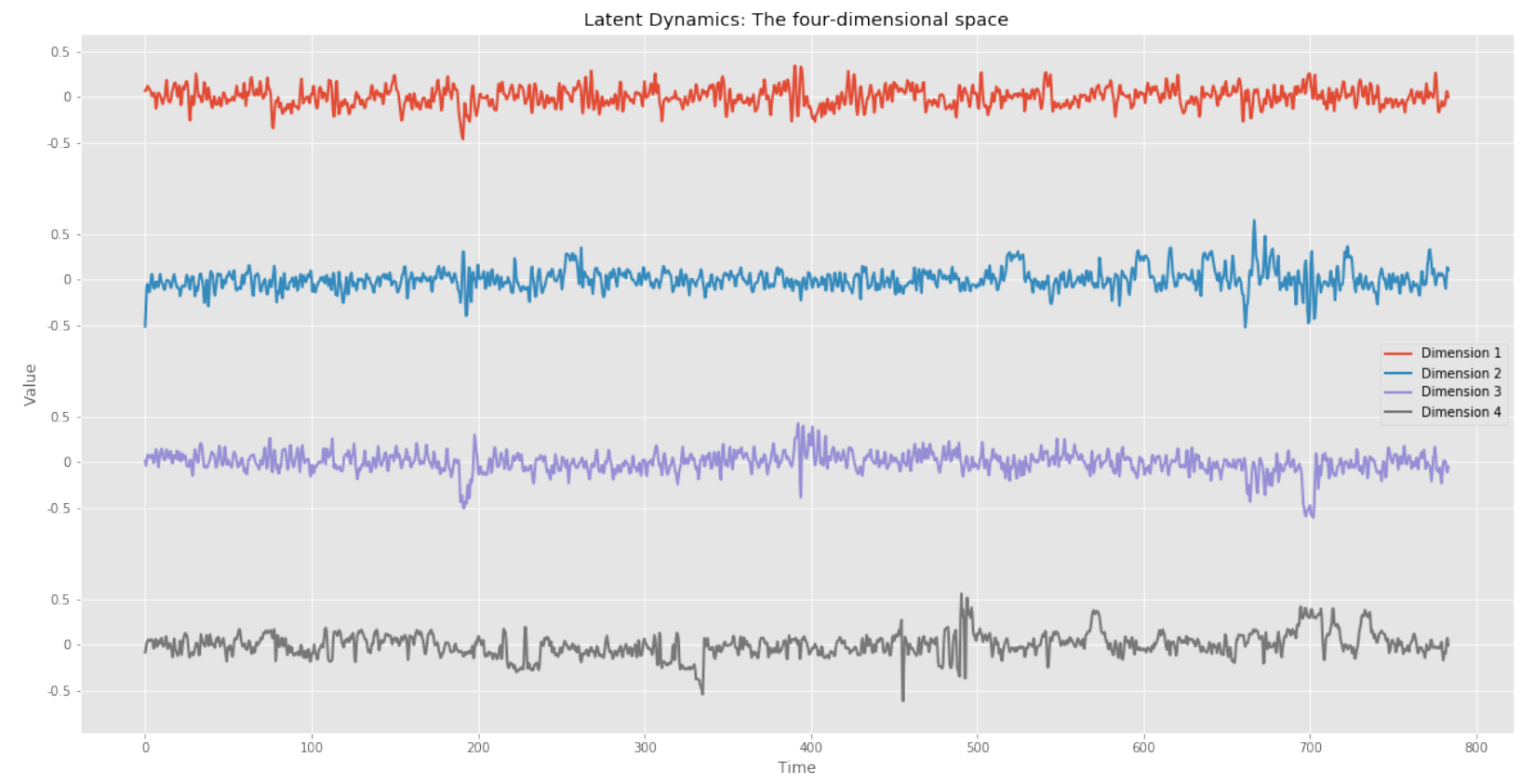

Figure 13: The estimated four-dimensional latent dynamics from the GPJM from the fMRI experiment. Latent dimensions are color-coded differently. GPJM: Gaussian process joint model. fMRI: functional magnetic resonance imaging. 

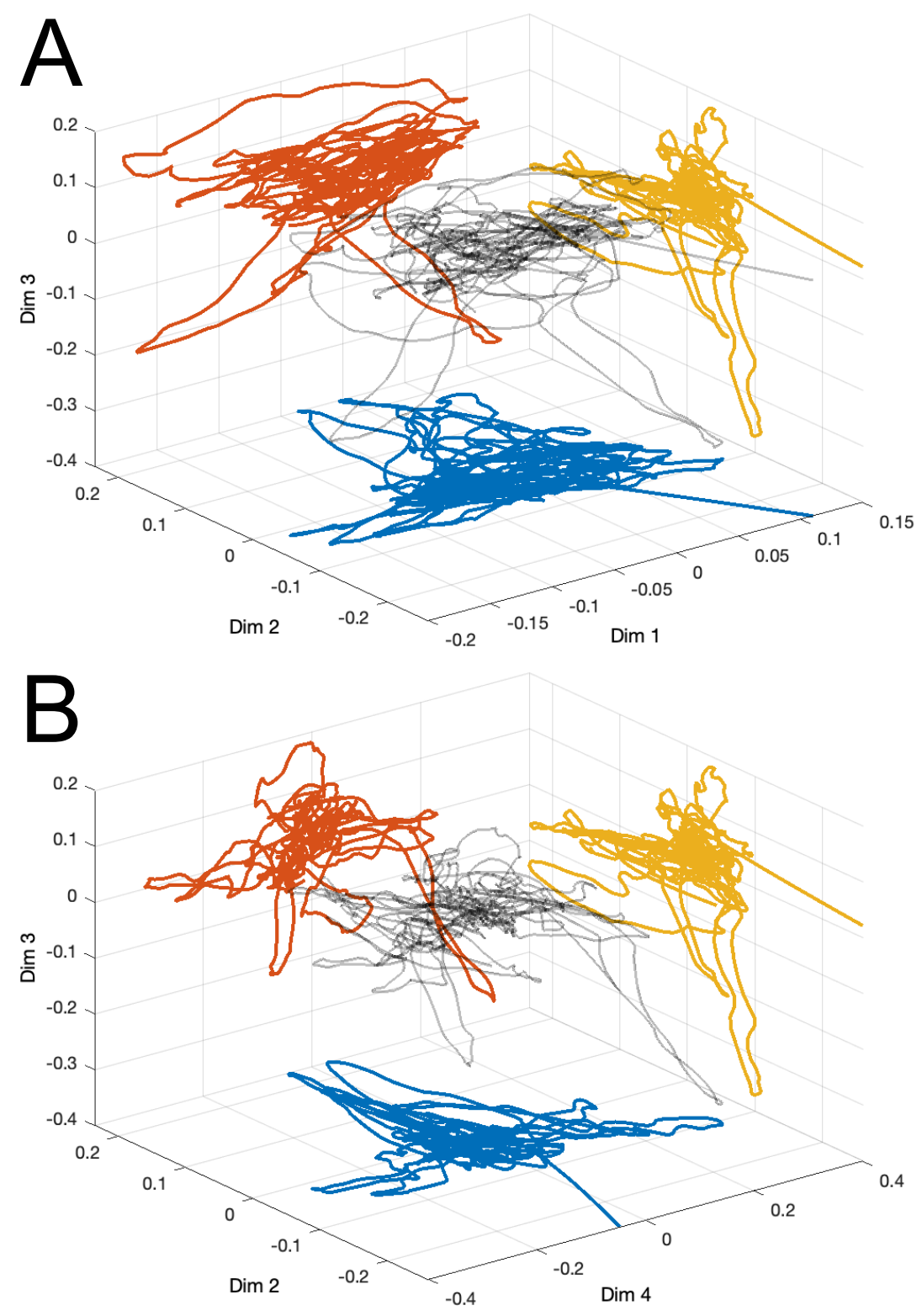

Figure 14: Three-dimensional plots of the four-dimensional latent dynamics estimated by the GPJM from the fMRI experiment. For visual clairty, the latent dynamics are filtered using the Savitzky-Golay filter with a window size of 51 and the third-order polynomial approximation. (A) Comparison of the Dimension 1 (x-axis), 2 (y-axis), and 3 (z-axis). A black line represents the three-dimensional trajectory of the dynamics generated by Dimensions 1, 2, and 3. Red, yellow, and blue lines are the two-dimensional projections of the dynamics trajectory generated by Dimensions 1 and 3 , 2 and 3, and 1 and 2, respectively. (B) Comparison of the Dimension 4 (x-axis), 2 (y-axis), and 3 (z-axis). A black line represents the three-dimensional trajectory of the dynamics generated by Dimensions 4, 2, and 3. Red, yellow, and blue lines are the two-dimensional projections of the dynamics trajectory generated by Dimensions 4 and 3,2 and 3, and 4 and 2, respectively. GPJM: Gaussian process joint model. fMRI: functional magnetic resonance imaging. 